\title{
A Family of Hyperbolic-Type Explicit Force Regulators with Active Velocity Damping for Robot Manipulators
}

\author{
Fernando Reyes-Cortés $\mathbb{D}^{\mathbb{D}},{ }^{1}$ César Chávez-Olivares $\mathbb{D}^{\mathbb{D}},{ }^{2}$ and Emilio J. González-Galván $\mathbb{D}^{3}$ \\ ${ }^{1}$ Facultad de Ciencias de la Electrónica, Benemérita Universidad Autónoma de Puebla, 72570 Puebla, PUE, Mexico \\ ${ }^{2}$ Departamento de Ingeniería Robótica, Universidad Autónoma de Aguascalientes, Campus Sur, \\ Av. Prolongación Mahatma Gandhi No. 6601, 20340 Aguascalientes, AGS, Mexico \\ ${ }^{3}$ Facultad de Ingeniería, Universidad Autónoma de San Luis Potosí, Av. Manuel Nava 8, Zona Universitaria, \\ 78290 San Luis Potosí, SLP, Mexico \\ Correspondence should be addressed to Fernando Reyes-Cortés; fernando.reyes@correo.buap.mx
}

Received 28 December 2017; Accepted 1 March 2018; Published 16 April 2018

Academic Editor: L. Fortuna

Copyright (C) 2018 Fernando Reyes-Cortés et al. This is an open access article distributed under the Creative Commons Attribution License, which permits unrestricted use, distribution, and reproduction in any medium, provided the original work is properly cited.

\begin{abstract}
This paper addresses the explicit force regulation problem for robot manipulators in interaction tasks. A new family of explicit force-control schemes is presented, which includes a term driven by a large class of saturated-type hyperbolic functions to handle the force error. Also, an active velocity damping term with the purpose of obtaining energy dissipation on the contact surface is incorporated plus compensation for gravity. In order to ensure asymptotic stability of the closed-loop system equilibrium point in Cartesian space, we propose a strict Lyapunov function. A force sensor placed at the end-effector of the robot manipulator is used in order to feed back the measure of the force error in the closed-loop, and an experimental comparison of the performance $\mathscr{L}_{2}$-norm between 5 explicit force control schemes, which are the classical proportional-derivative (PD), arctangent, and square-root controls and two members of the proposed control family, on a two-degree-of-freedom, direct-drive robot manipulator, is presented.
\end{abstract}

\section{Introduction}

The use of robotic systems has increased during the last four decades. Nowadays, several industrial applications continue to benefit from robotic systems in order to automate repetitive or hazardous maneuvers. It is well known that, in applications involving an automatic process in which the robot is moving freely within its workspace or executes tasks with no interaction with the environment, a control system based on position or motion control represents a viable alternative. However, in applications where the manipulator performs interaction tasks, the manipulator encounters environmental constraints and the interaction forces are not negligible [1-3].

In this situation, the use of motion-control strategies will produce an unsuccessful execution of the task; the adequate implementation of an interaction task requires, in addition to motion/position control strategies, force control and a detailed and accurate modeling of the system and the environment [4-9]. A particular type of interaction control is the impedance control scheme which can regulate environmental forces according to the specified mechanical impedance such as inertial, stiffness, and damping effects. Impedance control belongs to the category of indirect force control, which may not require measurements of contact forces; in addition, the user cannot specify a desired contact force [2, 4, 9-12].

In contrast, force sensing and force control are essential for the successful execution of tasks involving interaction between the manipulator and the environment. The explicit force control strategies are the most suitable option for controlling interaction forces between the robot manipulator and the environment. Such control schemes have a large number of applications in medical and industrial environments, as they are capable of increasing the speed and quality in applications such as deburring, grinding of surfaces, assembly processes, and other operations $[3,10,13-16]$. 
Some types of force control can be cited, such as [17] where two auxiliary, automatically controlled wings of a monohull ship used to attenuate the roll due to the waves are presented. These wings are variably dipped into the water in order to introduce a force that reduces the oscillations on the ship. Two different compensators for the roll effect have been designed, the first one using classical frequency domain techniques and the second being an adaptive, linear quadratic compensator with a multilayer perceptron neural network. Simulations and experimental tests performed on the ship are presented. In [18], an impedance function is proposed to specify a desired force directly in a robust robot force tracking impedance scheme, which employs a neural network as compensator to cancel out all uncertainties; simulation studies are presented. In [19], the robot dynamics are decomposed into force, position, and redundant joint subspaces; then, using that decomposition in a neural network-based adaptive control scheme for hybrid force/position in Lyapunov sense is proposed. Numerical simulation studies were performed in the model of a two-link, rigid robot manipulator. In [20], an approach based on hybrid force/position with fuzzy PID structure with unspecified robot dynamics in the presence of external disturbances, including experimental results on a PUMA-560 robot, is presented.

Unlike the impedance control schemes, the concept of explicit force control is to consider a desired force rather than the position or velocity references. Then the force error is defined as the difference between a desired force and the actual interaction force, measured with a force sensor mounted on the end-effector, enabling the possibility of ensuring force regulation. The explicit force control selects the directions in which the end-effector position should be controlled and those directions in which the forces controlled by the end-effector are applied on some environment, for a given task. This type of control strategies has two feedback loops for separated, position, and force control and is referred to as hybrid force/position control [1, 21-34].

The original hybrid force/position control concept was proposed in [1]; the basic idea is to divide the robot manipulator space into two orthogonal spaces using of a selection matrix. Position control and force control are applied on two spaces to achieve the capability of compliance control. Explicit force control involves the direct command and measurement of force values in order to control the contact force to a desired value.

Considering that explicit force control is an important topic as research area, it currently represents an open problem of interest for the scientific community and several research efforts have focused on this topic during the last four decades. As a result, different control schemes have been proposed in the literature; for example, we can cite the fundamental works $[1,5,24,26,32]$. Progressively this theme has been growing; for instance, [35] describes the design and implementation of a hybrid force/motion scheme to control the interaction force of a robot manipulator, using a PD controller with gain-scheduled linear parameter-varying controller; however, although the reference for this control scheme does not include a stability proof, it presents experimental results on a six-degree-of-freedom manipulator. The paper [36] proposes the design of a robust adaptive neural networkbased hybrid position/force control with PD-type structure for manipulators. It includes a stability analysis in the sense of Lyapunov, but only simulation results with a model of a two-link robot manipulator are presented. In [37], the robustness and stability of disturbance observer based on explicit linear force control systems are analyzed. The validity of the proposals is verified with experimental results on a linear $\mathrm{dc}$ motor.

In [38], explicit force regulators for robot manipulators are presented. The control structure includes a nonlinear term formed by square-root-type functions to drive the force error, with an active velocity damping plus compensation of gravity forces. The stability analysis is performed in Lyapunov sense; in order to obtain asymptotic stability, LaSalle's theorem is used and experimental results on a two-degree-of-freedom robot are presented. In [39], an optimization-based approach for the regulation of excessive or insufficient forces at the endeffector level is introduced. Their approach minimizes the interaction force error at the robot end-effector and considers the linear impedance model and penalty functions in order to handle the constraints on the interaction forces; stability and convergence properties are analyzed taking into account force limits. The proposed scheme is validated via experimental results for a robotic grasping task. The work [40] presents the state of the art on compliant control algorithms applied to both stiff and soft joints based on electric motors for robot manipulators; there is a widely used control structure for all the works presented in this survey, which is the simple PD control.

Recently, in [16] a double active observer control is proposed to control the desired interaction and motion compensation under contact with the heart, relying on robot force control techniques. In [34], a position-based force explicit control strategy based on online trajectory prediction, with proportional-integral-type control structure, is presented. In this work, the experimental results show that the proposed method has a good control effect on the contact force. In [41], the regulation of the clamping force of a robot manipulator gripper is analyzed and discussed, when applied to rescue activities in the ruins after disasters; for this problem, a hybrid force/position control on a cascade PID-scheme and fuzzy sliding mode is presented; the control algorithms were validated with experimental results, whose force errors were acceptable. Force trajectories during isometric force-matching task involving contractions are described in [42]; a PID control with different gains and frequencies is introduced to examine the nature of the contractions in many individuals, which vary substantially. This variability can be explained by discrete PID with varying model parameters to adjust the muscle commands. The position and force control task presented in [43] has been defined considering the manipulator and environment models; asymptotic stability of the control systems is demonstrated, and experimental study of the issue is presented.

Most of the previous works use explicit force control strategies with PD or PID schemes; however, in general, there is a lack of proof on asymptotic stability analysis with 
different control structures; only a limited number of works have proposed strict Lyapunov functions. Therefore, our main motivation is in the theoretical and practical aspects of the problem. The objective of this paper is to present the design and analysis of a new explicit force control family with a term driven by a large class of saturatedtype hyperbolic functions to handle the force error for the problem of force regulation in interaction tasks. This control structure includes also an active velocity damping term with the purpose of obtaining energy dissipation on the contact surface. To ensure global asymptotic stability of the closed-loop system equilibrium point in Cartesian space, we propose a strict Lyapunov function. Additionally, we present in this work another contribution, consisting of the mathematical development of a Cartesian dynamics model property (on the vector of centripetal and Coriolis forces in Cartesian space, see Appendix), which is useful for stability analysis.

The paper includes also an experimental comparison of the performance obtained between 5 explicit force control schemes; they are the classical proportional-derivative (PD), arctangent, square-root controls, and two members of the proposed control family on a two-degree-of-freedom, directdrive robot manipulator. A force sensor placed at the endeffector of the robot manipulator is used in order to feed back the measure of the force error in the closed-loop, which is given as the difference between a desired and actual force. The Cartesian explicit force control is converted to joint torque signal using the transpose Jacobian introduced by Takegaki and Arimoto [44].

The paper is organized as follows. In Section 2, the joint-space dynamic model of robot manipulators and its relationship with the Cartesian dynamic model are presented; it also describes the most important properties used in the stability analysis of the control schemes. In Section 3, the new family of explicit force regulators is presented, as well as the design of the strict function required to carry out the asymptotic stability analysis in Lyapunov sense. In Section 4, the experimental results on a two-degree-of-freedom, directdrive robot manipulator including a performance comparison of the control schemes under evaluation are presented. Finally, our conclusions are in Section 5.

\section{Dynamic Model of the Robot Manipulator}

It is a well-known fact that the dynamic model of a robot manipulator with rigid links is described by a secondorder system formed by nonlinear differential equations. This model describes a relationship between the generalized joint torques/forces applied by the actuators and the motion of the robot, which incorporates the position, velocity, and acceleration joints. In joint space, the dynamic model of a robot manipulator with $n$ degrees of freedom is defined by [32]

$$
\boldsymbol{\tau}=\mathbf{M}(\mathbf{q}) \ddot{\mathbf{q}}+\mathbf{C}(\mathbf{q}, \dot{\mathbf{q}}) \dot{\mathbf{q}}+\mathbf{g}(\mathbf{q})+\mathbf{f}(\dot{\mathbf{q}}),
$$

where $\mathbf{q}, \dot{\mathbf{q}}, \ddot{\mathbf{q}} \in \mathbb{R}^{n}$ denote the joint displacements, velocities, and acceleration values, respectively, $\boldsymbol{\tau} \in \mathbb{R}^{n \times 1}$ stands for the vector of generalized forces/torques applied, $\mathbf{M}(\mathbf{q}) \in \mathbb{R}^{n \times n}$ is the symmetric positive-definite inertia matrix, $\mathbf{C}(\mathbf{q}, \dot{\mathbf{q}}) \epsilon$ $\mathbb{R}^{n \times n}$ is the matrix of centripetal and Coriolis torques, $\mathbf{g}(\mathbf{q}) \in \mathbb{R}^{n}$ is the vector of gravitational torques, and, finally, $\mathbf{f}(\dot{\mathbf{q}}) \in \mathbb{R}^{n}$ is the friction torque vector. For analysis purposes, only the viscous-friction model is considered in this paper; that is, $\mathbf{f}(\dot{\mathbf{q}})=\mathbf{B} \dot{\mathbf{q}}$, where $\mathbf{B}$ is a diagonal matrix formed by the viscous-friction coefficients of each joint.

Moreover, the above model (1) can be expressed in terms of coordinates associated with a coordinate system attached to the tool by using a coordinate transformation $[6,25,45]$. For the formulation of the model in Cartesian space, it is assumed that the manipulator performs in a nonsingular configuration.

Let us consider the relationship between the joint velocities $\dot{\mathbf{q}}$ and the end-effector Cartesian velocities $\dot{\mathbf{x}}$, given by $\dot{\mathbf{x}}=\mathbf{J}(\mathbf{q}) \dot{\mathbf{q}}$, where $\mathbf{J}(\mathbf{q})$ is referred to as the analytical Jacobian matrix; that is, $\mathbf{J}(\mathbf{q})=\partial \mathbf{h}(\mathbf{q}) / \partial \mathbf{q} \in \mathbb{R}^{n \times n}$, where $\mathbf{h}(\mathbf{q})$ is the direct kinematic. A well-known relationship exists between the applied forces $\mathbf{f}_{\chi}$ on the end-effector and the applied torques $\tau$ in the joints, which is referred to as the Jacobian Transposed Controller [44]:

$$
\boldsymbol{\tau}=\mathbf{J}^{T}(\mathbf{q}) \mathbf{f}_{\chi}
$$
[45]

The Cartesian dynamic model of a robot manipulator is

$$
\mathbf{M}_{\chi} \ddot{\mathbf{x}}+\mathbf{C}_{\chi} \dot{\mathbf{x}}+\mathbf{g}_{\chi}+\mathbf{f}_{f \chi}=\mathbf{f}_{\chi}-\mathbf{f}_{e}
$$

where the term $\mathbf{f}_{e}$ represents the external forces applied to the end-effector, such as the forces appearing in an interaction task. The terms $\mathbf{M}_{\chi}, \mathbf{C}_{\chi}, \mathbf{g}_{\chi}$, and $\mathbf{f}_{f \chi}$ are described as

$$
\begin{aligned}
& \mathbf{M}_{\chi}=\mathbf{J}^{-T}(\mathbf{q}) \mathbf{M}(\mathbf{q}) \mathbf{J}^{-1}(\mathbf{q}) \\
& \mathbf{C}_{\chi}=\mathbf{J}^{-T}(\mathbf{q}) \mathbf{C}(\mathbf{q}, \dot{\mathbf{q}}) \mathbf{J}^{-1}(\mathbf{q})-\mathbf{M}_{\chi} \dot{\mathbf{J}}(\mathbf{q}) \mathbf{J}^{-1}(\mathbf{q}) \\
& \mathbf{g}_{\chi}=\mathbf{J}^{-T}(\mathbf{q}) \mathbf{g}(\mathbf{q}) \\
& \mathbf{f}_{f \chi}=\mathbf{B}_{\chi} \dot{\mathbf{x}}=\underbrace{\mathbf{J}^{-T}(\mathbf{q}) \mathbf{B J}^{-1}(\mathbf{q})}_{\mathbf{B}_{\chi}} \dot{\mathbf{x}} .
\end{aligned}
$$

We use the notation $\dot{\mathbf{J}}(\mathbf{q})$ to indicate the time derivative of the Jacobian matrix, $\dot{\mathbf{J}}(\mathbf{q})=(d / d t) \mathbf{J}(\mathbf{q})$, and the inverse of transpose Jacobian matrix is denoted by $\mathbf{J}^{-T}(\mathbf{q})=\left[\mathbf{J}^{-1}(\mathbf{q})\right]^{T}$.

Next, some well-known properties that are useful in the analysis and design of our controller scheme are presented.

Property 1. The inertia matrix $\mathbf{M}_{\chi}>0$ is symmetric and definite positive (on a workspace without singularity).

$$
\begin{gathered}
\mathbf{M}_{\chi}>0 \Longleftrightarrow \\
\mathbf{M}_{\chi}^{-1}>0 .
\end{gathered}
$$


Property 2. The matrix $\mathbf{M}_{\chi}$ is upper and lower bounded by

$$
\lambda_{\mathrm{m}}\left\{\mathbf{M}_{\chi}\right\} \mathbf{I} \leq \mathbf{M}_{\chi} \leq \lambda_{\mathrm{M}}\left\{\mathbf{M}_{\chi}\right\} \mathbf{I},
$$

where $\lambda_{\mathrm{M}}\left\{\mathbf{M}_{\chi}\right\}$ and $\lambda_{\mathrm{m}}\left\{\mathbf{M}_{\chi}\right\}$ stand for the maximum and minimum eigenvalues of matrix $\mathbf{M}_{\chi}$, respectively.

Property 3. $\dot{\mathbf{M}}_{\chi}=\mathbf{C}_{\chi}+\mathbf{C}_{\chi}^{T}$; the matrix $\dot{\mathbf{M}}_{\chi}-2 \mathbf{C}_{\chi}$ is skewsymmetric and it satisfies

$$
\frac{1}{2} \dot{\mathbf{x}}^{T}\left[\dot{\mathbf{M}}_{\chi}-2 \mathbf{C}_{\chi}\right] \dot{\mathbf{x}} \equiv 0
$$

Property 4. The norm of vector $\mathbf{C}_{\chi} \dot{\mathbf{x}}$ is bounded, as indicated below:

$$
\left\|\mathbf{C}_{\chi} \dot{\mathbf{x}}\right\| \leq k_{c x}\|\dot{\mathbf{x}}\|^{2}
$$

for some $k_{c x} \in \mathbb{R}_{+}$. As part of the contributions of this article, a brief demonstration of this property is included in the Appendix.

The following decoupled model is considered for modeling the compliant environment [3]:

$$
\mathbf{f}_{e}=\mathbf{K}_{e}\left(\mathbf{x}-\mathbf{x}_{e}\right),
$$

where $\mathbf{K}_{e}$ is a diagonal matrix referred to as the environment's stiffness matrix and the term $\mathbf{x}_{e}$ denotes the contact position with the environment, while $\mathbf{x}$ represents the actual position of the end-effector.

\section{A New Family of Explicit Force Regulator Schemes}

In this section, a new family of force control schemes with hyperbolic-type structure for robot manipulators is presented. Consider the following family with large class of explicit force control schemes plus gravity compensation, given by

$$
\mathbf{f}_{\chi}=\mathbf{K}_{p}\left[\begin{array}{c}
\frac{\cosh ^{k-1}\left(\tilde{f}_{1}\right) \sinh \left(\tilde{f}_{1}\right)}{1+\cosh ^{k}\left(\tilde{f}_{1}\right)} \\
\frac{\cosh ^{k-1}\left(\tilde{f}_{2}\right) \sinh \left(\tilde{f}_{2}\right)}{1+\cosh ^{k}\left(\tilde{f}_{2}\right)} \\
\vdots \\
\frac{\cosh ^{k-1}\left(\tilde{f}_{n}\right) \sinh \left(\tilde{f}_{n}\right)}{1+\cosh ^{k}\left(\tilde{f}_{n}\right)}
\end{array}\right]-\mathbf{K}_{v} \dot{\mathbf{x}}+\mathbf{g}_{\chi}+\mathbf{f}_{e},
$$

where $k \in \mathbb{N}_{+}$is a positive integer, $\mathbf{K}_{p}, \mathbf{K}_{v} \in \mathbb{R}^{n \times n}$ are the proportional and derivative gains, which are positive-definite diagonal matrices, and $\widetilde{\mathbf{f}}(\mathrm{t})$ is the force error vector, which is defined as the difference between a desired force $\mathbf{f}_{d}$ and the actual force $\mathbf{f}(t)$ on the end-effector, measured from the force sensor; $\widetilde{\mathbf{f}}(t)=\mathbf{f}_{d}-\mathbf{f}(t)$. The applied torques on the robot joints are derived from (2); that is, $\boldsymbol{\tau}=\mathbf{J}^{T}(\mathbf{q}) \mathbf{f}_{\chi}$.

The first term in the proposed control scheme (10) represents a large class of hyperbolic-type regulators to drive the force error; this one is derived from energy shaping of the artificial potential energy [44]. The second term acts as an active velocity damping; the next component includes the Cartesian gravity forces $\mathbf{g}_{\chi}$; for implementation purposes, partial knowledge of these gravity forces is required, and the last term $\mathbf{f}_{e}$ represents the interaction forces.

The closed-loop equation expressed in state variables $\left[\begin{array}{ll}\widetilde{\mathbf{f}} & \dot{\mathbf{x}}\end{array}\right]^{T} \in \mathbb{R}^{2 n}$ is obtained by combining the dynamics model (3) and control law (10):

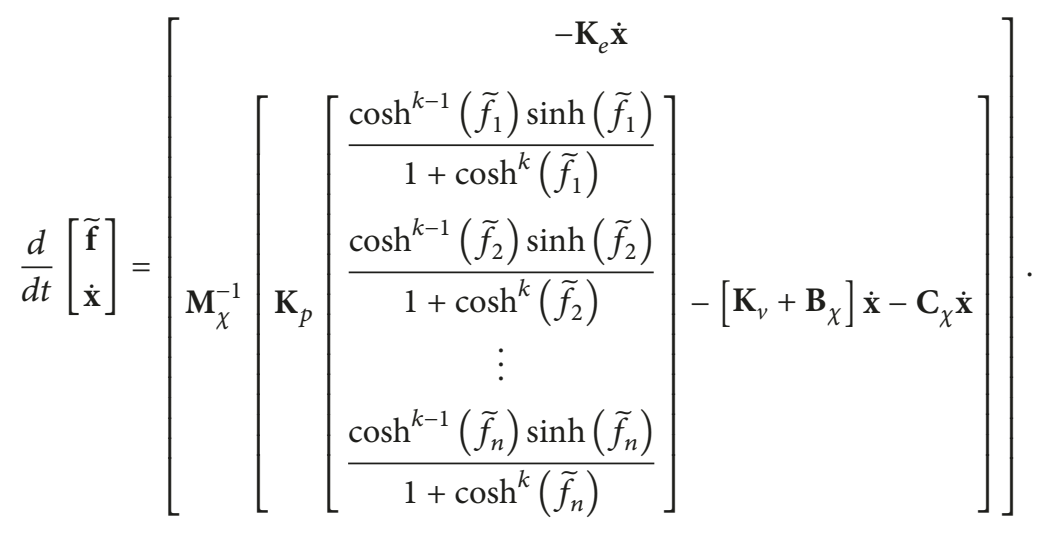

In order to demonstrate that the state origin is an equilibrium point, it is necessary to consider the Jacobian matrix $\mathbf{J}(\mathbf{q})$ with full rank; for example, $\operatorname{rank}[\mathbf{J}(\mathbf{q})]=n$; in other words, the Cartesian dynamic model requires a singularityfree workspace. The following assumptions are taken into account: 
(a) The Jacobian matrix $\mathbf{J}(\mathbf{q})$ is full rank and its spectral norm $\|\mathbf{J}(\mathbf{q})\|<k_{J}$ is bounded for all $\mathbf{q} \in \mathbb{R}^{n}$, where $k_{J}$ is a positive constant, $k_{J} \in \mathbb{R}_{+}$.

(b) According to the environment's stiffness and friction models, the stiffness $\mathbf{K}_{e}$ and viscous-friction $\mathbf{B}_{\chi}$ matrices are diagonal and positive-definite matrices, respectively; then $\left[\mathbf{K}_{e}+\mathbf{B}_{\chi}\right] \dot{\mathbf{x}}=\mathbf{0} \Leftrightarrow \dot{\mathbf{x}}=\mathbf{0}$.

The first component of the closed-loop equation (11) satisfies $-\mathbf{K}_{e} \dot{\mathbf{x}}=\mathbf{0} \in \mathbb{R}^{n} \Leftrightarrow \dot{\mathbf{x}}=\mathbf{0} \in \mathbb{R}^{n}$, due to the fact that the matrix $\mathbf{K}_{e}$ is a diagonal definite positive matrix. For the second component in (11), we use Property 1 of the inertia matrix; then the first term inside the brackets composed of the proportional gain $\mathbf{K}_{p}$ and the vector of hyperbolic functions results in the vector $\mathbf{0} \in \mathbb{R}^{n} \Leftrightarrow \widetilde{\mathbf{f}}=\mathbf{0} \in \mathbb{R}^{n}$, since $\mathbf{K}_{p}$ is a positive-definite matrix and each component $\cosh ^{k-1}\left(\tilde{f}_{i}\right) \sinh \left(\tilde{f}_{i}\right) /\left(1+\cosh ^{k}\left(\tilde{f}_{i}\right)\right)=0 \Leftrightarrow \sinh \left(\tilde{f}_{i}\right)=$ $0 \Leftrightarrow \widetilde{f}_{i}=0, \forall k \in \mathbb{N}_{+}$, and $i=1, \ldots, n$. We have also $\mathbf{C}_{\chi} \dot{\mathbf{x}}=0$ if $\dot{\mathbf{x}}=\mathbf{0} \in \mathbb{R}^{n}$ and $\mathbf{C}_{\chi} \neq \mathbf{0} \in \mathbb{R}^{n \times n}$ in the singularity-free workspace. Therefore, the closed-loop system (11) is autonomous differential equation and the state space origin exists and it is a local equilibrium point.

3.1. Control Problem Statement. The problem of explicit force control consists of finding a Cartesian control scheme $\mathbf{f}_{\chi}$ such that the force error vector $\widetilde{\mathbf{f}}(t)$ and Cartesian velocity $\dot{\mathbf{x}}(t)$ converge asymptotically stably to equilibrium point of the closedloop equation (11) at least for sufficiently small initial conditions $[\widetilde{\mathbf{f}}(0), \dot{\mathbf{x}}(0)]^{T}$; then the following objective is satisfied:

$$
\lim _{t \rightarrow \infty}\left[\begin{array}{c}
\widetilde{\mathbf{f}}(t) \\
\dot{\mathbf{x}}(t)
\end{array}\right]=\mathbf{0} \in \mathbb{R}^{2 n} .
$$

3.2. Proposition. Consider the closed-loop system (11), under conditions sufficiently small; then the equilibrium point $[\widetilde{\mathbf{f}}(t) \dot{\mathbf{x}}(t)] \in \mathbb{R}^{2 n}$ is locally asymptotically stable and the control objective (12) is achieved.

Proof. To carry out the local stability analysis for the dynamic system (11), we use Lyapunov's direct method through the following strict Lyapunov function (a strict Lyapunov function is a positive-definite function, whose time derivative along the trajectories of closed-loop system yields a negativedefinite function), which is composed by the sum of the robot manipulator kinetic and artificial potential energy, and a cross-term between force error $\widetilde{\mathbf{f}}$ and velocity $\dot{\mathbf{x}}$ :

$$
\begin{aligned}
V(\dot{\mathbf{x}}, \widetilde{\mathbf{f}})= & \frac{1}{2} \dot{\mathbf{x}}^{T} \mathbf{M}_{\chi} \dot{\mathbf{x}}+\mathcal{U}_{a}\left(\mathbf{K}_{p}, \mathbf{K}_{e}^{-1}, \widetilde{\mathbf{f}}\right) \\
& -\frac{\varepsilon_{0}}{1+\|\tilde{\mathbf{f}}\|} \widetilde{\mathbf{f}}^{T} \mathbf{M}_{\chi} \dot{\mathbf{x}},
\end{aligned}
$$

where $\varepsilon_{0}$ is a positive constant number defined in the following interval:

$$
\begin{aligned}
& \min \left\{\sqrt{\frac{\rho^{\prime} \lambda_{\mathrm{m}}\left\{\mathbf{K}_{e}^{-1} \mathbf{K}_{p}\right\}}{k \lambda_{\mathrm{M}}\left\{\mathbf{M}_{\chi}\right\}}, \frac{\lambda_{\mathrm{m}}\left\{\mathbf{K}_{v}\right\}+\lambda_{\mathrm{m}}\left\{\mathbf{B}_{\chi}\right\}+k_{c x}}{2 \lambda_{\mathrm{M}}\left\{\mathbf{K}_{e}\right\} \lambda_{\mathrm{M}}\left\{\mathbf{M}_{\chi}\right\}},}\right. \\
& \left.\frac{\lambda_{\mathrm{m}}\left\{\mathbf{K}_{p}\right\}\left[\lambda_{\mathrm{m}}\left\{\mathbf{K}_{v}\right\}+\lambda_{\mathrm{m}}\left\{\mathbf{B}_{\chi}\right\}+k_{c x}\right]}{2 \lambda_{\mathrm{m}}\left\{\mathbf{K}_{p}\right\} \lambda_{\mathrm{M}}\left\{\mathbf{K}_{e}\right\} \lambda_{\mathrm{M}}\left\{\mathbf{M}_{\chi}\right\}+(1 / 4)\left[\lambda_{\mathrm{m}}\left\{\mathbf{K}_{v}\right\}+\lambda_{\mathrm{m}}\left\{\mathbf{B}_{\chi}\right\}\right]^{2}}\right\} \\
& >\varepsilon_{0}>0,
\end{aligned}
$$

where $\rho^{\prime}$ is a positive constant, $\lambda_{\mathrm{m}}\left\{\mathbf{K}_{e}^{-1} \mathbf{K}_{p}\right\}$ is the minimum eigenvalue of the product $\mathbf{K}_{e}^{-1} \mathbf{K}_{p}$, the minimum eigenvalue of the environment's inverse stiffness matrix is $\lambda_{\mathrm{m}}\left\{\mathbf{K}_{e}^{-1}\right\}$, and $\lambda_{\mathrm{M}}\left\{\mathbf{K}_{p}\right\}, \lambda_{\mathrm{M}}\left\{\mathbf{K}_{v}\right\}, \lambda_{\mathrm{M}}\left\{\mathbf{B}_{\chi}\right\}$ are the maximum eigenvalues of the proportional and derivative gains and viscous-friction matrix, respectively. It is important to note that the constant $\varepsilon_{0}$ is only required for analysis purposes and its numerical value is not required for the control scheme.

The artificial potential energy function $\boldsymbol{U}_{a}\left(\mathbf{K}_{p}, \mathbf{K}_{e}^{-1}, \widetilde{\mathbf{f}}\right)$ is a continuously differentiable and positive-definite function, given by

$$
\begin{aligned}
& \mathscr{U}_{a}\left(\mathbf{K}_{p}, \mathbf{K}_{e}^{-1}, \tilde{\mathbf{f}}\right) \\
& =\frac{1}{k}\left[\begin{array}{c}
\sqrt{\ln \left(1+\cosh ^{k}\left(\tilde{f}_{1}\right)\right)-\ln (2)} \\
\sqrt{\ln \left(1+\cosh ^{k}\left(\tilde{f}_{2}\right)\right)-\ln (2)} \\
\vdots \\
\sqrt{\ln \left(1+\cosh ^{k}\left(\tilde{f}_{n}\right)\right)-\ln (2)}
\end{array}\right] \\
& \cdot \mathbf{K}_{p} \mathbf{K}_{e}^{-1}\left[\begin{array}{c}
\sqrt{\ln \left(1+\cosh ^{k}\left(\tilde{f}_{1}\right)\right)-\ln (2)} \\
\sqrt{\ln \left(1+\cosh ^{k}\left(\tilde{f}_{2}\right)\right)-\ln (2)} \\
\vdots \\
\sqrt{\ln \left(1+\cosh ^{k}\left(\tilde{f}_{n}\right)\right)-\ln (2)}
\end{array}\right] .
\end{aligned}
$$

The candidate Lyapunov function (13) corresponds to the total energy of the closed-loop (11) and can be rewritten as

$$
\begin{aligned}
V(\dot{\mathbf{x}}, \tilde{\mathbf{f}})= & \frac{1}{2}\left[\dot{\mathbf{x}}-\frac{\varepsilon_{0}}{1+\|\tilde{\mathbf{f}}\|} \widetilde{\mathbf{f}}\right]^{T} \mathbf{M}_{\chi}\left[\dot{\mathbf{x}}-\frac{\varepsilon_{0}}{1+\|\widetilde{\mathbf{f}}\|} \widetilde{\mathbf{f}}\right] \\
& +\mathscr{U}_{a}\left(\mathbf{K}_{p}, \mathbf{K}_{e}^{-1}, \tilde{\mathbf{f}}\right)-\frac{1}{2} \frac{\varepsilon_{0}^{2}}{[1+\|\tilde{\mathbf{f}}\|]^{2}} \widetilde{\mathbf{f}}^{T} \mathbf{M}_{\chi} \widetilde{\mathbf{f}} .
\end{aligned}
$$

Now, we will give sufficient conditions to make $V(\dot{\mathbf{x}}, \widetilde{\mathbf{f}})$ a positive-definite function. The first term of the right side of (16) is a definite positive function with respect to $\widetilde{\mathbf{f}}$ and $\dot{\mathbf{x}}$, because inertia matrix $\mathbf{M}_{\chi}$ is definite positive. Next, we provide upper and lower bounds on the second and third terms of the Lyapunov function (16). 
The artificial energy function (15) has a unique and global minimum at $\widetilde{\mathbf{f}}=\mathbf{0} \in \mathbb{R}^{n}$, and it is not a decreasing function; then there exists positive constant $\rho^{\prime} \in \mathbb{R}_{+}$, such that $(1 / k) \rho^{\prime} \lambda_{\mathrm{m}}\left\{\mathbf{K}_{e}^{-1} \mathbf{K}_{p}\right\}\|\widetilde{\mathbf{f}}\|^{2} \leq \mathcal{U}_{a}\left(\mathbf{K}_{p}, \mathbf{K}_{e}^{-1}, \widetilde{\mathbf{f}}\right)$. Therefore, the second and third terms of function (16) satisfy

$$
\begin{aligned}
& {\left[\frac{1}{k} \rho^{\prime} \lambda_{\mathrm{m}}\left\{\mathbf{K}_{e}^{-1} \mathbf{K}_{p}\right\}-\varepsilon_{0}^{2} \lambda_{\max }\left\{\mathbf{M}_{\chi}\right\}\right]\|\tilde{\mathbf{f}}\|^{2}} \\
& \leq \frac{1}{k}\left\|\left[\begin{array}{c}
\ln \left(1+\cosh ^{k}\left(\tilde{f}_{1}\right)\right)-\ln (2) \\
\ln \left(1+\cosh ^{k}\left(\tilde{f}_{2}\right)\right)-\ln (2) \\
\vdots \\
\ln \left(1+\cosh ^{k}\left(\tilde{f}_{n}\right)\right)-\ln (2)
\end{array}\right]\right\| \lambda_{\min }\left\{\mathbf{K}_{p} \mathbf{K}_{e}^{-1}\right\} \\
& -\varepsilon_{0}^{2} \frac{\|\widetilde{\mathbf{f}}\|^{2}}{\left[1+\|\tilde{\mathbf{f}}\|^{2}\right.} \lambda_{\max }\left\{\mathbf{M}_{\chi}\right\} \\
& \leq \mathscr{U}_{a}\left(\mathbf{K}_{p}, \mathbf{K}_{e}^{-1}, \widetilde{\mathbf{f}}\right)-\frac{1}{2} \frac{\varepsilon_{0}^{2}}{[1+\|\widetilde{\mathbf{f}}\|]^{2}} \widetilde{\mathbf{f}}^{T} \mathbf{M}_{\chi} \widetilde{\mathbf{f}} .
\end{aligned}
$$

On the left-hand side of the inequality (17), to ensure that $\left[(1 / k) \rho^{\prime} \lambda_{\mathrm{m}}\left\{\mathbf{K}_{e}^{-1} \mathbf{K}_{p}\right\}-\varepsilon_{0}^{2} \lambda_{\max }\left\{\mathbf{M}_{\chi}\right\}\right] \quad$ is a positive expression, $\varepsilon_{0}$ must be in the interval $0<\varepsilon_{0}<$ $\sqrt{\rho^{\prime} \lambda_{\mathrm{m}}\left\{\mathbf{K}_{e}^{-1} \mathbf{K}_{p}\right\} / k \lambda_{\max }\left\{\mathbf{M}_{\chi}\right\}}$, which is satisfied by the first term of (14). Hence, the Lyapunov function $V(\dot{\mathbf{x}}, \widetilde{\mathbf{f}})$ given by (13) is a definite positive function in local form.

The total derivative of the candidate Lyapunov function (13) with respect to time is

$$
\begin{aligned}
& \dot{V}(\dot{\mathbf{x}}, \widetilde{\mathbf{f}})=\dot{\mathbf{x}}^{T} \mathbf{M}_{\chi} \ddot{\mathbf{x}}+\frac{1}{2} \dot{\mathbf{x}}^{T} \dot{\mathbf{M}}_{\chi} \dot{\mathbf{x}} \\
& -\left[\begin{array}{c}
\frac{\cosh ^{k-1}\left(\tilde{f}_{1}\right) \sinh \left(\tilde{f}_{1}\right)}{1+\cosh ^{k}\left(\tilde{f}_{1}\right)} \\
\frac{\cosh ^{k-1}\left(\tilde{f}_{2}\right) \sinh \left(\tilde{f}_{2}\right)}{1+\cosh ^{k}\left(\tilde{f}_{2}\right)} \\
\vdots \\
\frac{\cosh ^{k-1}\left(\tilde{f}_{n}\right) \sinh \left(\tilde{f}_{n}\right)}{1+\cosh ^{k}\left(\tilde{f}_{n}\right)}
\end{array}\right]^{T} \mathbf{K}_{p} \mathbf{K}_{e}^{-1} \underbrace{\mathbf{K}_{e} \dot{\mathbf{x}}}_{\tilde{\mathbf{f}}} \\
& -\frac{\varepsilon_{0}}{1+\|\widetilde{\mathbf{f}}\|} \widetilde{\mathbf{f}}^{T} \mathbf{M}_{\chi} \ddot{\mathbf{x}}-\frac{\varepsilon_{0}}{1+\|\widetilde{\mathbf{f}}\|} \widetilde{\mathbf{f}}^{T} \underbrace{\dot{\mathbf{M}}_{\chi}}_{\mathbf{C}_{\chi}^{T}+\mathbf{C}_{\chi}} \dot{\mathbf{x}} \\
& +\frac{\varepsilon_{0}}{1+\|\tilde{\mathbf{f}}\|} \underbrace{\dot{\mathbf{x}}^{T}}_{\dot{\mathbf{f}}^{T}} \mathbf{K}_{e} \mathbf{M}_{\chi} \dot{\mathbf{x}} \\
& -\varepsilon_{0} \frac{\overbrace{\dot{\mathbf{x}}^{T} \mathbf{K}_{e}}^{\dot{\mathbf{f}}^{T}} \widetilde{\mathbf{f}}}{\|\widetilde{\mathbf{f}}\|[1+\|\tilde{\mathbf{f}}\|]^{2}} \widetilde{\mathbf{f}}^{T} \mathbf{M}_{\chi} \dot{\mathbf{x}} .
\end{aligned}
$$

Substituting the closed-loop equation (11), we also use Property 3 ; then it yields,

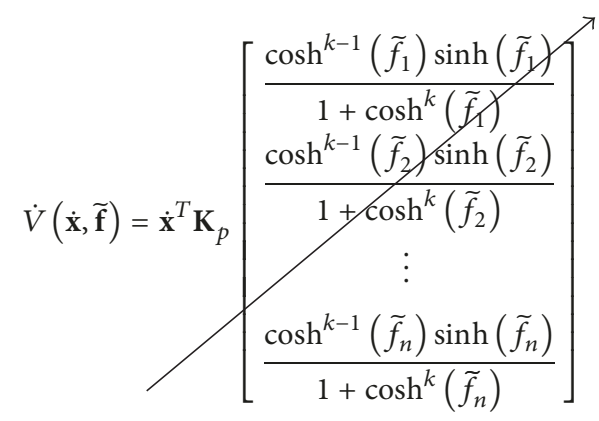

$$
-\dot{\mathbf{x}}^{T}\left[\mathbf{K}_{v}+\mathbf{B}_{\chi}\right] \dot{\mathbf{x}}^{T}-\dot{\mathbf{x}}^{T} \mathbf{C}_{\mathcal{X}^{\mathbf{x}}}{ }^{T}+\frac{1}{2} \dot{\mathbf{x}}^{T} \dot{\mathbf{M}}_{\chi} \dot{\mathbf{x}}
$$

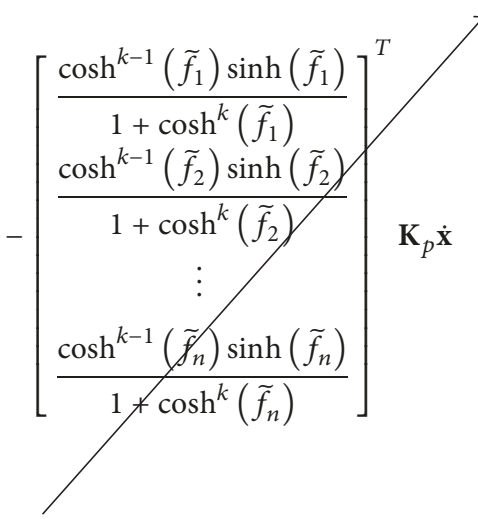

$$
\begin{aligned}
& -\frac{\varepsilon_{0}}{1+\|\tilde{\mathbf{f}}\|} \widetilde{\mathbf{f}}^{T} \mathbf{K}_{p}\left[\begin{array}{c}
\frac{\cosh ^{k-1}\left(\tilde{f}_{1}\right) \sinh \left(\tilde{f}_{1}\right)}{1+\cosh ^{k}\left(\tilde{f}_{1}\right)} \\
\frac{\cosh ^{k-1}\left(\tilde{f}_{2}\right) \sinh \left(\tilde{f}_{2}\right)}{1+\cosh ^{k}\left(\tilde{f}_{2}\right)} \\
\vdots \\
\frac{\cosh ^{k-1}\left(\tilde{f}_{n}\right) \sinh \left(\tilde{f}_{n}\right)}{1+\cosh ^{k}\left(\tilde{f}_{n}\right)}
\end{array}\right] \\
& +\frac{\varepsilon_{0}}{1+\|\widetilde{\tilde{f}}\|} \widetilde{\mathbf{f}}^{T}\left[\mathbf{K}_{v}+\mathbf{B}_{\chi}\right] \dot{\mathbf{x}}+\frac{\varepsilon_{0}}{1+\|\tilde{\mathbf{f}}\|} \tilde{\mathbf{f}}^{T} \mathscr{\phi}_{\chi} \dot{\mathbf{x}} \\
& -\frac{\varepsilon_{0}}{1+\|\widetilde{\mathbf{f}}\|} \widetilde{\mathbf{f}}^{T}\left[\mathbf{C}_{\chi}^{T}+\mathscr{Q}_{\chi}\right] \dot{\mathbf{x}}+\underbrace{\frac{\varepsilon_{0}}{1+\|\widetilde{\mathbf{f}}\|}}_{\varepsilon_{0} /(1+\|\tilde{\mathbf{f}}\|) \leq \varepsilon_{0}} \dot{\mathbf{x}}^{T} \mathbf{K}_{e} \mathbf{M}_{\chi} \dot{\mathbf{x}} \\
& -\varepsilon_{0} \frac{\dot{\mathbf{x}}^{T} \mathbf{K}_{e} \widetilde{\mathbf{f}}}{\|\tilde{\mathbf{f}}\|[1+\|\tilde{\mathbf{f}}\|]^{2}} \widetilde{\mathbf{f}}^{T} \mathbf{M}_{\chi} \dot{\mathbf{x}},
\end{aligned}
$$

where, after cancelling some terms, we obtain

$$
\dot{V}(\dot{\mathbf{x}}, \widetilde{\mathbf{f}})=-\dot{\mathbf{x}}^{T}\left[\mathbf{K}_{v}+\mathbf{B}_{\chi}-\varepsilon_{0} \mathbf{K}_{e} \mathbf{M}_{\chi}\right] \dot{\mathbf{x}}^{T}
$$




$$
\begin{aligned}
& -\frac{\varepsilon_{0}}{1+\|\widetilde{\mathbf{f}}\|} \widetilde{\mathbf{f}}^{T} \mathbf{K}_{p}\left[\begin{array}{c}
\frac{\cosh ^{k-1}\left(\tilde{f}_{1}\right) \sinh \left(\tilde{f}_{1}\right)}{1+\cosh ^{k}\left(\tilde{f}_{1}\right)} \\
\frac{\cosh ^{k-1}\left(\tilde{f}_{2}\right) \sinh \left(\tilde{f}_{2}\right)}{1+\cosh ^{k}\left(\tilde{f}_{2}\right)} \\
\vdots \\
\frac{\cosh ^{k-1}\left(\tilde{f}_{n}\right) \sinh \left(\tilde{f}_{n}\right)}{1+\cosh ^{k}\left(\tilde{f}_{n}\right)}
\end{array}\right] \\
& +\frac{\varepsilon_{0}}{1+\|\widetilde{\mathbf{f}}\|} \widetilde{\mathbf{f}}^{T}\left[\mathbf{K}_{v}+\mathbf{B}_{\chi}\right] \dot{\mathbf{x}} \\
& -\quad \underbrace{\frac{\varepsilon_{0}}{1+\|\tilde{\mathbf{f}}\|} \widetilde{\mathbf{f}}^{T} \mathbf{C}_{\chi}^{T} \dot{\mathbf{x}}} \\
& \left(\varepsilon_{0} /(1+\|\tilde{\mathbf{f}}\|)\right)\|\tilde{\mathbf{f}}\|\left\|\mathbf{C}_{x} \dot{\dot{x}}\right\| \leq \varepsilon_{0} k_{c x}\|\| \dot{\mathbf{x}} \|^{2} \\
& -\varepsilon_{0} \frac{\dot{\mathbf{x}}^{T} \mathbf{K}_{e} \tilde{\mathbf{f}}}{\|\tilde{\mathbf{f}}\|[1+\|\tilde{\mathbf{f}}\|]^{2}} \widetilde{\mathbf{f}}^{T} \mathbf{M}_{\chi} \dot{\mathbf{x}} .
\end{aligned}
$$

Note that the second term of the right side in (20) corresponds to the gradient of the artificial potential energy $(\partial / \partial \widetilde{\mathbf{f}}) \mathcal{U}_{a}\left(\mathbf{K}_{p}, \mathbf{K}_{e}^{-1}, \widetilde{\mathbf{f}}\right)$; it is a vector composed of saturated functions with hyperbolic-type structure, for each $i$ th component $\cosh ^{k-1}\left(\widetilde{f}_{i}\right) \sinh \left(\widetilde{f}_{i}\right) /\left(1+\cosh ^{k}\left(\widetilde{f}_{i}\right)\right)=\left(\cosh ^{k}\left(\widetilde{f}_{i}\right) /\right.$ $\left.\left(1+\cosh ^{k}\left(\widetilde{f}_{i}\right)\right)\right)\left(\sinh \left(\widetilde{f}_{i}\right) / \cosh \left(\widetilde{f}_{i}\right)\right)=\left(\cosh ^{k}\left(\widetilde{f}_{i}\right) /(1+\right.$ $\left.\left.\cosh ^{k}\left(\tilde{f}_{i}\right)\right)\right) \tanh \left(\tilde{f}_{i}\right)$; note also that $1+\cosh ^{k}\left(\tilde{f}_{i}\right)>\cosh ^{k}\left(\tilde{f}_{i}\right)$ $\Leftrightarrow \quad\left(\cosh ^{k}\left(\tilde{f}_{i}\right) /\left(1+\cosh ^{k}\left(\tilde{f}_{i}\right)\right)\right)<1$; then $-1 \leq$ $\cosh ^{k-1}\left(\tilde{f}_{i}\right) \sinh \left(\tilde{f}_{i}\right) /\left(1+\cosh ^{k}\left(\tilde{f}_{i}\right)\right) \leq 1$; there exist positive constants $\rho_{2}, \rho_{2}^{\prime} \in \mathbb{R}_{+}$, such that $\rho_{2}^{\prime}\left|\tilde{f}_{i}\right|$ $\leq\left(\cosh ^{k-1}\left(\tilde{f}_{i}\right) \sinh \left(\tilde{f}_{i}\right) /\left(1+\cosh ^{k}\left(\tilde{f}_{i}\right)\right)\right) \tilde{f}_{i} \leq \rho_{2}\left|\tilde{f}_{i}\right|$; therefore it satisfies

$$
\rho_{2}^{\prime}\|\widetilde{\mathbf{f}}\|^{2}<\widetilde{\mathbf{f}}^{T} \frac{\partial}{\partial \widetilde{\mathbf{f}}} \mathcal{U}_{a}\left(\mathbf{K}_{p}, \mathbf{K}_{e}^{-1}, \widetilde{\mathbf{f}}\right)<\rho_{2}\|\widetilde{\mathbf{f}}\|^{2},
$$

$\forall \widetilde{\mathbf{f}} \neq \mathbf{0} \in \mathbb{R}^{n}$.
In other words, the second term of function (20) satisfies the following condition:

$$
\begin{aligned}
& \frac{\varepsilon_{0}}{1+\|\widetilde{\mathbf{f}}\|}\|\widetilde{\mathbf{f}}\|^{2} \lambda_{\mathrm{m}}\left\{\mathbf{K}_{p}\right\} \\
& \leq \frac{\varepsilon_{0}}{1+\|\widetilde{\mathbf{f}}\|} \widetilde{\mathbf{f}}^{T} \mathbf{K}_{p}\left[\begin{array}{c}
\frac{\cosh ^{k-1}\left(\tilde{f}_{1}\right) \sinh \left(\tilde{f}_{1}\right)}{1+\cosh ^{k}\left(\tilde{f}_{1}\right)} \\
\frac{\cosh ^{k-1}\left(\tilde{f}_{2}\right) \sinh \left(\tilde{f}_{2}\right)}{1+\cosh ^{k}\left(\tilde{f}_{2}\right)} \\
\vdots \\
\frac{\cosh ^{k-1}\left(\tilde{f}_{n}\right) \sinh \left(\tilde{f}_{n}\right)}{1+\cosh ^{k}\left(\tilde{f}_{n}\right)}
\end{array}\right] \\
& \leq \frac{\varepsilon_{0}}{1+\|\widetilde{\mathbf{f}}\|}\|\widetilde{\mathbf{f}}\|^{2} \lambda_{\mathrm{M}}\left\{\mathbf{K}_{p}\right\},
\end{aligned}
$$

where $\lambda_{\mathrm{m}}\left\{\mathbf{K}_{p}\right\}$ and $\lambda_{\mathrm{M}}\left\{\mathbf{K}_{p}\right\}$ stand for the maximum and minimum eigenvalues of matrix $\mathbf{K}_{p}$, respectively. Using Property 4 , the fourth term from (23) satisfies $-\left(\varepsilon_{0} /(1+\right.$ $\|\widetilde{\mathbf{f}}\|)) \widetilde{\mathbf{f}}^{T} \mathbf{C}_{\chi}^{T} \dot{\mathbf{x}} \leq\left(\varepsilon_{0} /(1+\|\widetilde{\mathbf{f}}\|)\right)\|\tilde{\mathbf{f}}\|\left\|\mathbf{C}_{\chi} \dot{\mathbf{x}}\right\| \leq \varepsilon_{0} k_{c x}\|\dot{\mathbf{x}}\|^{2}$. Next, by taking (22) and Cartesian dynamic model property (2), the expression for $\dot{V}(\dot{\mathbf{x}}, \widetilde{\mathbf{f}})(20)$ takes the form

$$
\begin{aligned}
& \dot{V}(\dot{\mathbf{x}}, \tilde{\mathbf{f}}) \\
& \leq-\left[\lambda_{\min }\left\{\mathbf{K}_{v}+\mathbf{B}_{\chi}\right\}+k_{c x}-\varepsilon_{0} \lambda_{\mathrm{M}}\left\{\mathbf{K}_{e} \mathbf{M}_{\chi}\right\}\right]\|\dot{\boldsymbol{x}}\|^{2} \\
& -\frac{\varepsilon_{0}}{1+\|\tilde{\mathbf{f}}\|} \lambda_{\mathrm{m}}\left\{\mathbf{K}_{p}\right\}\|\tilde{\mathbf{f}}\|^{2}+\underbrace{\frac{\varepsilon_{0}}{1+\|\tilde{\mathbf{f}}\|}}_{\varepsilon_{0} /(1+\|\tilde{\mathbf{f}}\|) \leq \varepsilon_{0}}\left[\lambda_{\mathrm{m}}\left\{\mathbf{K}_{v}+\mathbf{B}_{\chi}\right\}\right]\|\tilde{\mathbf{f}}\|\|\dot{\mathbf{x}}\|
\end{aligned}
$$

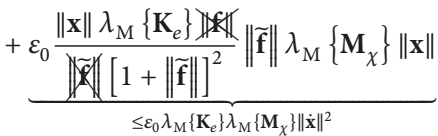

$$
\begin{aligned}
& \leq-\left[\lambda_{\text {min }}\left\{\mathbf{K}_{v}\right\}+\lambda_{\min }\left\{\mathbf{B}_{\chi}\right\}+k_{c x}-2 \varepsilon_{0} \lambda_{\mathrm{M}}\left\{\mathbf{K}_{e}\right\} \lambda_{\mathrm{M}}\left\{\mathbf{M}_{\chi}\right\}\right]\|\dot{\mathbf{x}}\|^{2} \\
& -\frac{\varepsilon_{0}}{1+\|\widetilde{\mathbf{f}}\|} \lambda_{\mathrm{m}}\left\{\mathbf{K}_{p}\right\}\|\tilde{\mathbf{f}}\|^{2}+\varepsilon_{0}\left[\lambda_{\mathrm{m}}\left\{\mathbf{K}_{v}\right\}+\lambda_{\mathrm{m}}\left\{\mathbf{B}_{\chi}\right\}\right]\|\tilde{\mathbf{f}}\|\|\dot{\mathbf{x}}\| .
\end{aligned}
$$

The time derivative of the Lyapunov function becomes

$$
\dot{V}(\dot{\mathbf{x}}, \tilde{\mathbf{f}}) \leq-\left[\begin{array}{c}
\|\dot{\mathbf{x}}\| \\
\|\tilde{\mathbf{f}}\|
\end{array}\right]^{T} \underbrace{\left[\begin{array}{cc}
{\left[\lambda_{\min }\left\{\mathbf{K}_{v}\right\}+\lambda_{\min }\left\{\mathbf{B}_{\chi}\right\}+k_{c x}-2 \varepsilon_{0} \lambda_{\mathrm{M}}\left\{\mathbf{K}_{e}\right\} \lambda_{\mathrm{M}}\left\{\mathbf{M}_{\chi}\right\}\right]} & -\frac{\varepsilon_{0}}{2}\left[\lambda_{\mathrm{m}}\left\{\mathbf{K}_{v}\right\}+\lambda_{\mathrm{m}}\left\{\mathbf{B}_{\chi}\right\}\right] \\
-\frac{\varepsilon_{0}}{2}\left[\lambda_{\mathrm{m}}\left\{\mathbf{K}_{v}\right\}+\lambda_{\mathrm{m}}\left\{\mathbf{B}_{\chi}\right\}\right] & \frac{\varepsilon_{0}}{1+\|\tilde{\mathbf{f}}\|} \lambda_{\mathrm{m}}\left\{\mathbf{K}_{p}\right\}
\end{array}\right]}_{\mathbf{Q}}\left[\begin{array}{l}
\|\dot{\mathbf{x}}\| \\
\|\widetilde{\mathbf{f}}\|
\end{array}\right]<0 .
$$

In order to ensure that matrix $\mathbf{Q}$ is positive definite, the number $\varepsilon_{0}$ must satisfy the second condition of the left side of (14); then the element $Q_{11}>0$; also the third condition of the left side in the expression (14) must be satisfied; then the determinant of matrix $\mathbf{Q}$ is positive. Considering that $\varepsilon_{0}$ satisfies those considerations, we can conclude that $\dot{V}(\dot{\mathbf{x}}, \widetilde{\mathbf{f}})$ is a negative-definite function; note that, due to the fact that both conditions $V(\dot{\mathbf{x}}, \widetilde{\mathbf{f}})>0$ and $\dot{V}(\dot{\mathbf{x}}, \widetilde{\mathbf{f}})<0$ are satisfied, the proposed Lyapunov function is a strict Lyapunov function. Therefore, according to Lyapunov's direct method, we conclude local asymptotic stability of the origin $\left[\begin{array}{ll}\widetilde{\mathbf{f}}^{T} & \dot{\mathbf{x}}^{T}\end{array}\right]^{T}=$ $\mathbf{0} \in \mathbb{R}^{2 n}$ of the closed-loop equation (11), which means that both state variables $\widetilde{\mathbf{f}}(t)$ and $\dot{\mathbf{x}}(t)$ asymptotically converge to zero, as $t \rightarrow \infty$. 


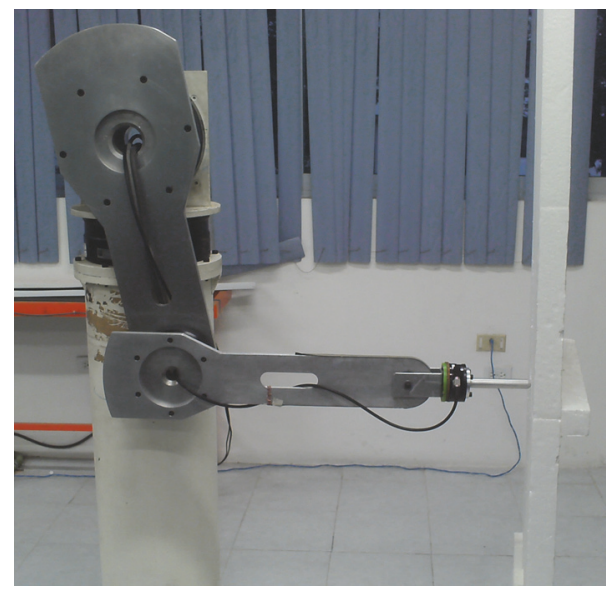

FIGURE 1: Experimental robot "Rotradi."

\section{Experimental Results}

The experimental results were obtained by using an experimental platform known as "Rotradi" [46], shown in Figure 1. This platform, designed and built at the Robotics Laboratory of Benemérita Universidad Autónoma de Puebla (BUAP) in Mexico, is a two-degree-of-freedom, direct-drive robot. Rotradi consists of two 6061 aluminum links, actuated by brushless, direct-drive servo actuators, in order to drive the joints without a gear reduction. The motors used in Rotradi are DM-1150A and DM-1015B models from Parker Compumotor, for the shoulder and elbow joints, respectively. Since the servomotors are operated in torque mode, they act as a torque source; in this mode, an analog voltage is used as a reference of the torque signal, in proportional scale. The features of the servomotors are shown in Table 1. A motion-control board of Precision MicroDynamics Inc. is included in the robot system; this device is designed for reading the encoders and generating reference voltages. The control algorithms, written in $\mathrm{C}$ code, run in real time with a 2.5 ms sample period, on a Pentium computer.

With the purpose of performing force sensing, a sixaxis force/torque sensor model ATI FT Gamma SI-130-10 is mounted at the end-effector of the robot manipulator. The sensor is capable of measuring force in the range of $\pm 130 \mathrm{~N}$ and torque within the range of $\pm 10 \mathrm{Nm}$. The sensor is connected to a $3.6 \mathrm{GHz}$ Pentium IV computer, in which the signals are processed using a Visual $\mathrm{C}++$ application. The communication between computers (for robot control and forcesensor readings) was enabled by using a communication protocol based on interruption signals, sent via parallel port.

The complete dynamic model for our experimental robot is reported in [46]. However, the gravitational torques vector and the Jacobian matrix are written in an explicit form in order to explain the experiments performed in this paper. The gravitational torques vector is given by

$$
\begin{aligned}
g_{1}(\mathbf{q})= & 54.813 \sin \left(q_{1}\right)-1.182 \cos \left(q_{1}\right) \\
& +2.145 \sin \left(q_{1}+q_{2}\right)
\end{aligned}
$$

$$
\begin{gathered}
+0.089 \cos \left(q_{1}+q_{2}\right) \\
g_{2}(\mathbf{q})=2.145 \sin \left(q_{1}+q_{2}\right)+0.089 \cos \left(q_{1}+q_{2}\right)
\end{gathered}
$$

while the Jacobian matrix is given as follows:

$$
\begin{aligned}
& \mathbf{J}(\mathbf{q}) \\
& =\left[\begin{array}{ll}
0.45 \cos \left(q_{1}\right)+0.69 \cos \left(q_{1}+q_{2}\right) & 0.69 \cos \left(q_{1}+q_{2}\right) \\
0.45 \sin \left(q_{1}\right)+0.69 \sin \left(q_{1}+q_{2}\right) & 0.69 \sin \left(q_{1}+q_{2}\right)
\end{array}\right] .
\end{aligned}
$$

We present an experimental comparison of five explicit force regulators on the two-degree-of-freedom, direct-drive robot manipulator. The evaluated schemes are the simple proportional-derivative (PD), denoted as $\mathbf{f}_{\chi_{\mathrm{PD}}}$, (arctangent) Atan and (square-root) Sqrt control schemes [38], represented as $\mathbf{f}_{\chi_{\text {Atan }}}$ and $\mathbf{f}_{\chi_{\text {Sqrt }}}$, respectively, and two hyperbolictype members of the proposed control family, denoted by $\mathbf{f}_{\chi_{1}}$ and $\mathbf{f}_{\chi_{2}}$, for exponents $k=1$ and $k=2$, respectively.

The structure of these regulators, evaluated on the twodegree-of-freedom robot manipulator, is given by

$$
\begin{aligned}
& \mathbf{f}_{\chi \mathrm{PD}}=\mathbf{K}_{p}\left[\begin{array}{c}
\tilde{f}_{x} \\
\tilde{f}_{y}
\end{array}\right]-\mathbf{K}_{v}\left[\begin{array}{c}
\dot{x}_{x} \\
\dot{x}_{y}
\end{array}\right]+\mathbf{g}_{\chi}+\mathbf{f}_{e}
\end{aligned}
$$

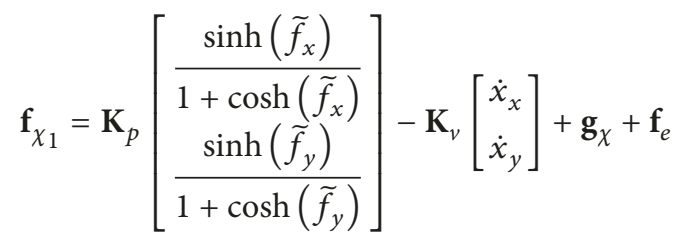

$$
\begin{aligned}
& \mathbf{f}_{\chi_{2}}=\mathbf{K}_{p}\left[\begin{array}{c}
\frac{\cosh \left(\tilde{f}_{x}\right) \sinh \left(\tilde{f}_{x}\right)}{1+\cosh ^{2}\left(\tilde{f}_{x}\right)} \\
\frac{\cosh \left(\tilde{f}_{y}\right) \sinh \left(\tilde{f}_{y}\right)}{1+\cosh ^{2}\left(\tilde{f}_{y}\right)}
\end{array}\right]-\mathbf{K}_{v}\left[\begin{array}{c}
\dot{x}_{x} \\
\dot{x}_{y}
\end{array}\right]+\mathbf{g}_{\chi} \\
& +\mathbf{f}_{e} \\
& \mathbf{f}_{\chi_{\text {Atan }}}=\mathbf{K}_{p}\left[\begin{array}{l}
\operatorname{atan}\left(\tilde{f}_{x}\right) \\
\operatorname{atan}\left(\tilde{f}_{y}\right)
\end{array}\right]-\mathbf{K}_{v}\left[\begin{array}{c}
\operatorname{atan}\left(\dot{x}_{x}\right) \\
\operatorname{atan}\left(\dot{x}_{y}\right)
\end{array}\right]+\mathbf{g}_{\chi}+\mathbf{f}_{e} \\
& \mathbf{f}_{\chi_{\mathrm{Sqrt}}}=\mathbf{K}_{p}\left[\begin{array}{c}
\frac{\tilde{f}_{x}}{\sqrt{1+\tilde{f}_{x}^{2}}} \\
\frac{\tilde{f}_{y}}{\sqrt{1+\tilde{f}_{y}^{2}}}
\end{array}\right]-\mathbf{K}_{v}\left[\begin{array}{c}
\frac{\dot{x}_{x}}{\sqrt{1+\dot{x}_{x}^{2}}} \\
\frac{\dot{x}_{y}}{\sqrt{1+\dot{x}_{y}^{2}}}
\end{array}\right]+\mathbf{g}_{\chi}+\mathbf{f}_{e} \text {. }
\end{aligned}
$$

All evaluated control schemes (27)-(31) have the following desired force references $\mathbf{f}_{d}=\left[\begin{array}{ll}f_{x d} & f_{y d}\end{array}\right]^{T}=\left[\begin{array}{ll}5.0 & 0.1\end{array}\right]^{T}[\mathrm{~N}]$; and the force error signals are given as $\tilde{\mathbf{f}}=\left[\begin{array}{ll}\tilde{f}_{x} & \tilde{f}_{y}\end{array}\right]^{T}=$ $\left[\begin{array}{ll}f_{x d}-f_{x} & f_{y d}-f_{y}\end{array}\right]^{T}$, while the gain matrices $\mathbf{K}_{p}$ and $\mathbf{K}_{v}$ are tuned in order to prevent saturation of the actuators, which is a complicated and time-consuming process where several 


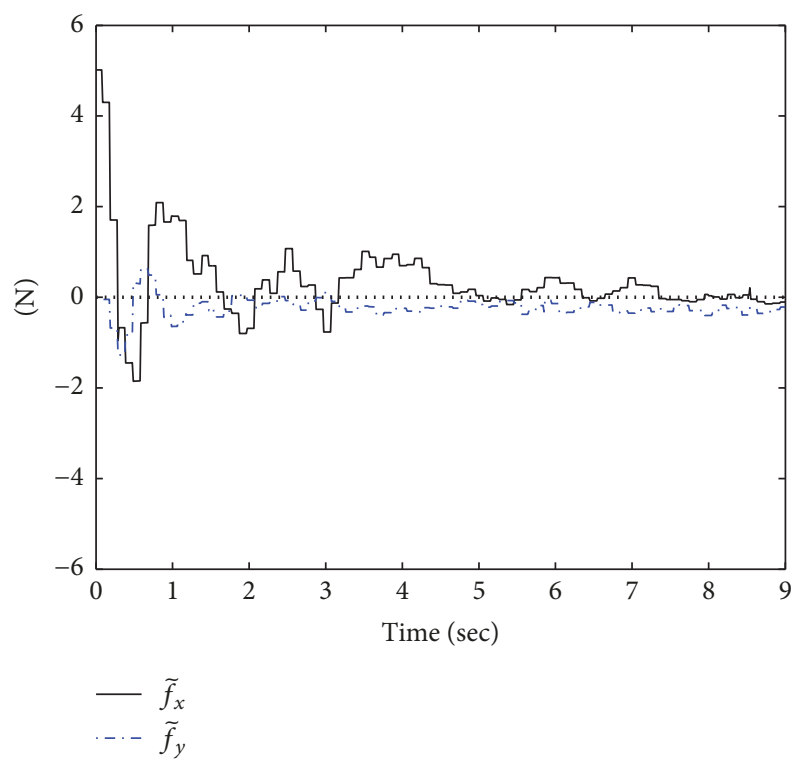

(a) Force error for the PD control $\mathbf{f}_{\chi \mathrm{PD}}$

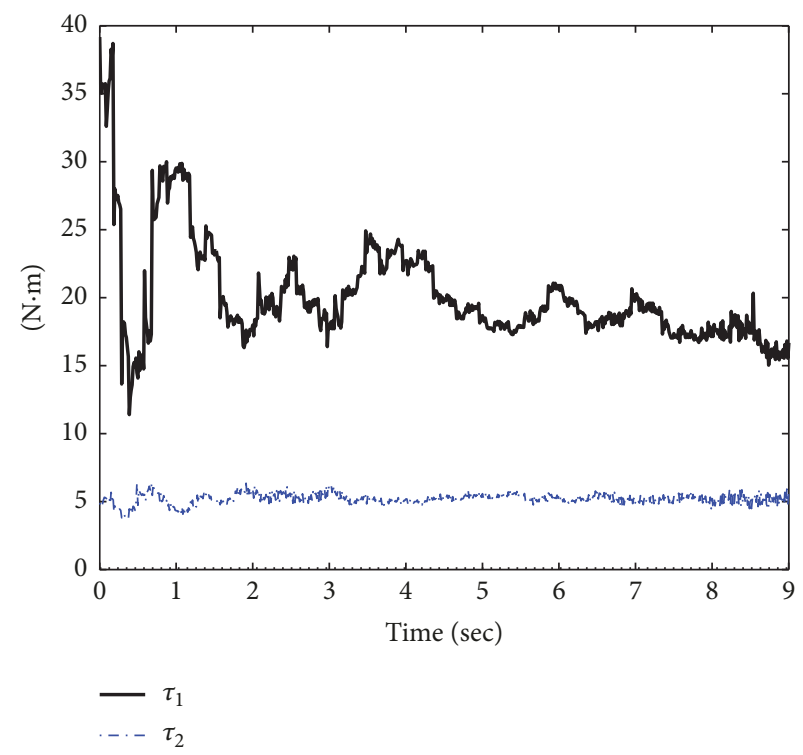

(b) Applied torques of the PD scheme $\boldsymbol{\tau}_{\mathrm{PD}}=\mathbf{J}(\mathbf{q})^{T} \mathbf{f}_{\chi_{\mathrm{PD}}}$

FIGURE 2: Experimental results for the PD control $\mathbf{f}_{\chi_{\mathrm{PD}}}$.

TABLE 1: Robot arm servo actuators

\begin{tabular}{lccc}
\hline Joint & Model & Max. torque [Nm] & Resolution [cpr] \\
\hline (1) Shoulder & DM-1150A & 150 & $1,024,000$ \\
(2) Elbow & DM-1015B & 15 & $1,024,000$ \\
\hline
\end{tabular}

trials were necessary in order to ensure a small transient state and overshoot and smaller steady-state error. The tuning used for each evaluated force control scheme is presented in Table 2. The hyperbolic structure in (28) and (29) has two arguments: one of them is the force error $\widetilde{\mathbf{f}}$, measured in Newtons; the second one is a constant with unit value (for simplicity, it does not appear in these equations), measured in Newton ${ }^{-1}$, so that the whole argument is dimensionless.

In order to test the performance of these control structures, an interaction experiment is designed; the experiment consists of placing the robot at an initial position (the force sensor attached to the tool at the end of the robot manipulator is placed at the contact position with the environment through a tangent-hyperbolic position control [47]), and the second link is normal to the plane of the wall. Once positioned, the tool attached to the force sensor is located close to the wall, allowing a slight touch on the surface. For this experiment, it is not necessary to control the end-effector orientation. The initial conditions of the force-regulation experiment are depicted in Figure 1.

Now, the experimental evaluation of the three controllers is described. The force error and the applied torques from PD control $\mathbf{f}_{\chi \mathrm{PD}}$ are presented in Figure 2. The force error signals for the PD control corresponding to two components $f_{x}$ and $f_{y}$ are depicted in Figure 2(a), which presents convergence properties. It can be noted that each component has a short transient $(4.5 \mathrm{sec})$, and both directions tend asymptotically to small neighborhood of zero; the steady-state force errors at time $t=9 \mathrm{sec}$ have small values, specifically $\left[\tilde{f}_{x}(t), \tilde{f}_{y}(t)\right]^{T}=$ $[0.2,0.25]^{T}$ N. Figure 2(b) describes the applied control torques $\boldsymbol{\tau}_{\mathrm{PD}}=\mathbf{J}(\mathbf{q})^{T} \mathbf{f}_{\chi_{\mathrm{PD}}}$ at the shoulder and elbow joints for the PD scheme. Note that the two torque signals are within the range of operation (see Table 1).

In Figure 3, the results for force error and applied torque of the Atan control are presented. Note that the experimental curves for both force error components $\left[\tilde{f}_{x}, \tilde{f}_{y}\right]^{T}$ converge to zero; the transitory response has small oscillations; for this control, its transient phase $(t=4.5 \mathrm{sec})$ is evolved for a shorter time than transient response of the PD control; the steady-state force errors at time $t=9 \mathrm{sec}$ have small values, $\left[\widetilde{f}_{1}(t), \tilde{f}_{2}(t)\right]^{T}=[0.18,0.29]^{T} \mathrm{~N}$, and the control algorithm $\mathbf{f}_{\chi_{\text {Atan }}}$ achieves the force-regulation objective. The torque signals for shoulder and elbow joints avoid saturation of the actuators.

Figure 4 describes the experimental results for the Sqrt control scheme; the transitory response is approximately $4.5 \mathrm{sec}$; the force error signals reach the steady-state error $\left[\tilde{f}_{x}(t), \tilde{f}_{y}(t)\right]^{T}=[-0.35,-0.31]^{T} \mathrm{~N}$, and the applied torques $\mathbf{J}(\mathbf{q})^{T} \mathbf{f}_{\chi_{\text {Sqrt }}}$ clearly evolve inside the prescribed limits shown in Table 1.

The force error and applied torque signals for the hyperbolic controller $\mathbf{f}_{\chi_{1}}$ with $k=1$ are shown in Figure 5. The experimental results for the force errors $\widetilde{\mathbf{f}}$ are depicted in Figure 5(a), which evolve until reaching steady-state errors 
TABLE 2: Gain matrices $\mathbf{K}_{p}$ and $\mathbf{K}_{v}$ for the force regulators.

\begin{tabular}{lcr}
\hline Regulator & $\mathbf{K}_{p}$ & $\mathbf{K}_{v}$ \\
\hline Proportional & $\operatorname{diag}(16.0,5.0) \operatorname{dimensionless}$ & $\operatorname{diag}(450.0,150.0) \mathrm{N}-\mathrm{sec} / \mathrm{m}$ \\
Hyperbolic, $k=1$ & $\operatorname{diag}(65.0,5.0) \mathrm{N}$ & $\operatorname{diag}(650.0,150.0) \mathrm{N}-\mathrm{sec} / \mathrm{m}$ \\
Hyperbolic, $k=2$ & $\operatorname{diag}(55.0,5.0) \mathrm{N}$ & $\operatorname{diag}(700.0,150.0) \mathrm{N}-\mathrm{sec} / \mathrm{m}$ \\
Atan & $\operatorname{diag}(30.0,5.0) \mathrm{N}$ & $\operatorname{diag}(400.0,150.0) \mathrm{N}$ \\
Sqrt & $\operatorname{diag}(39.0,5.0) \mathrm{N}$ & $\operatorname{diag}(400.0,150.0) \mathrm{N}$ \\
\hline
\end{tabular}

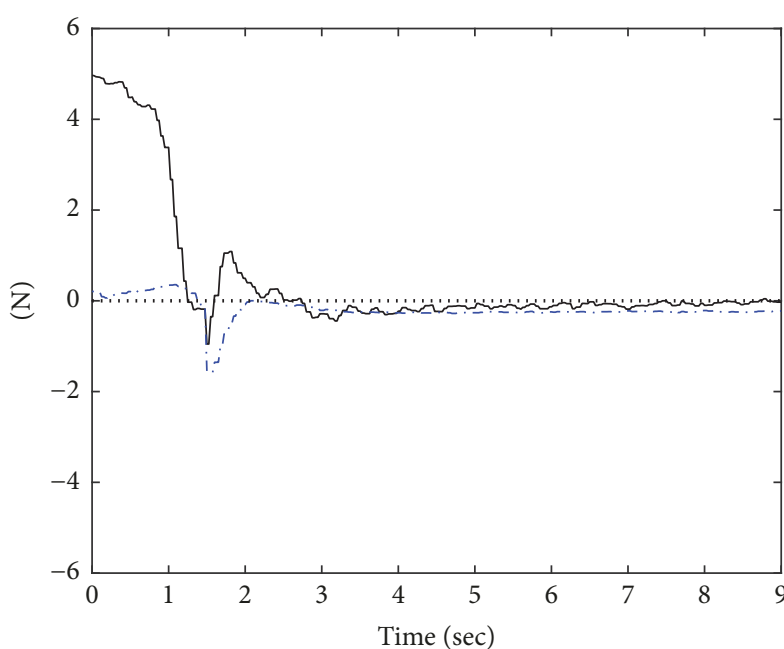

$-\tilde{f}_{x}$

$\cdot-\cdot \tilde{f}_{y}$

(a) Force error for the Atan controller

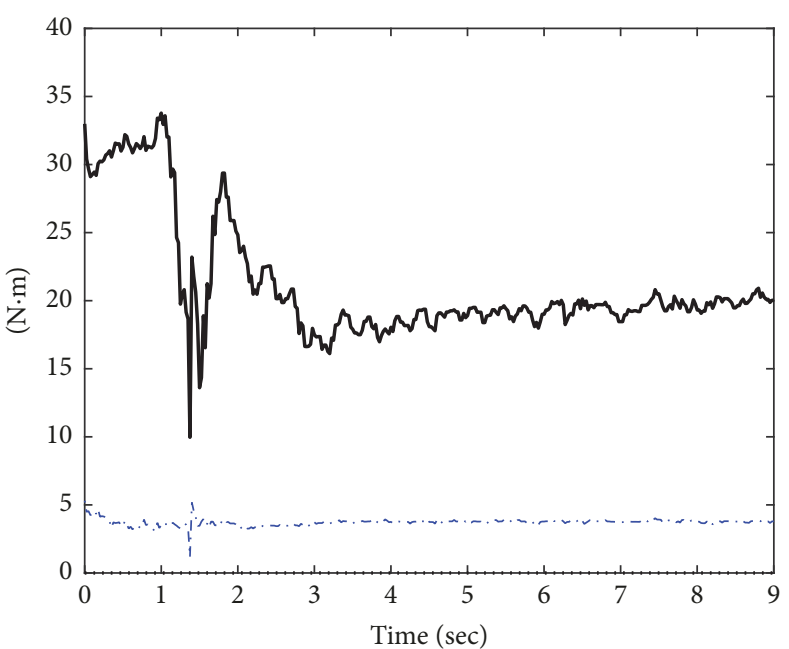

$-\tau_{1}$

(b) Applied Atan control torques

FIGURE 3: Experimental results for the Atan control $\boldsymbol{\tau}_{\text {Atan }}=\mathbf{J}(\mathbf{q})^{T} \mathbf{f}_{\chi_{\text {Atan }}}$.

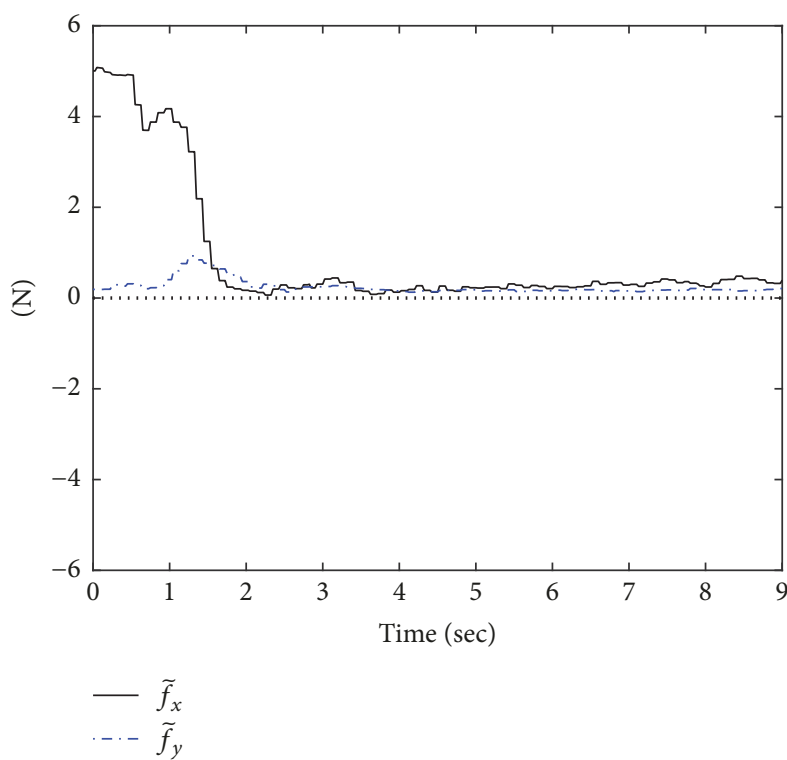

(a) Force error for the Sqrt controller

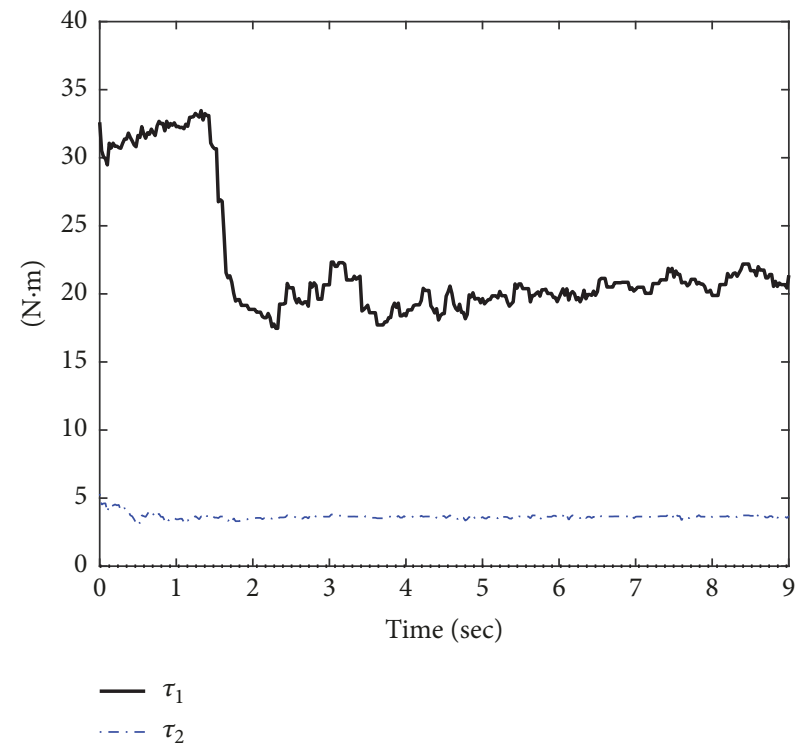

(b) Applied Sqrt control torques

FIGURE 4: Experimental results for the Sqrt control $\boldsymbol{\tau}_{\text {Sqrt }}=\mathbf{J}(\mathbf{q})^{T} \mathbf{f}_{\chi_{\text {Sqrt }}}$. 


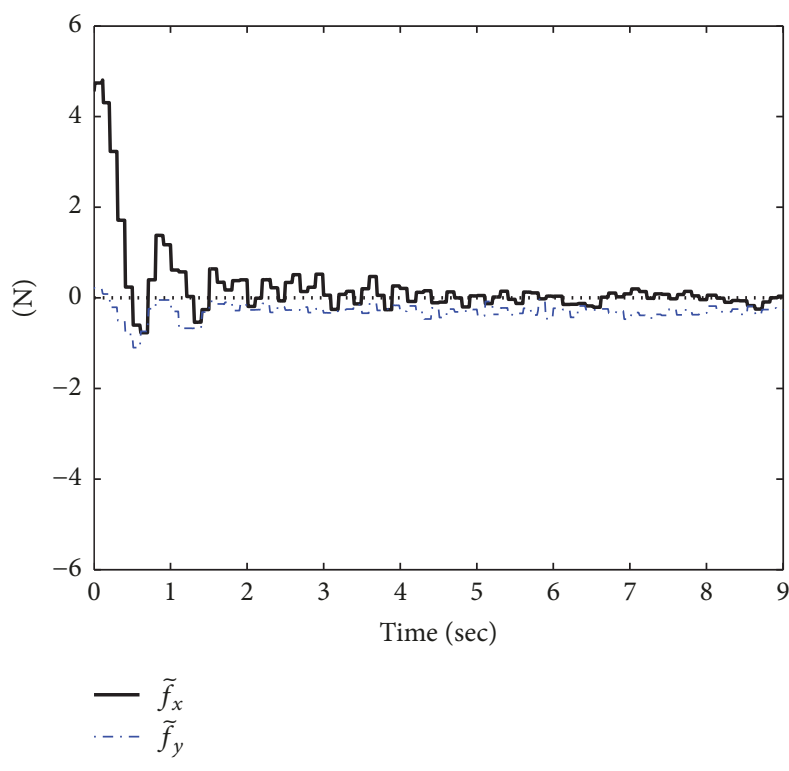

(a) Force error for the hyperbolic control scheme with $k=1$

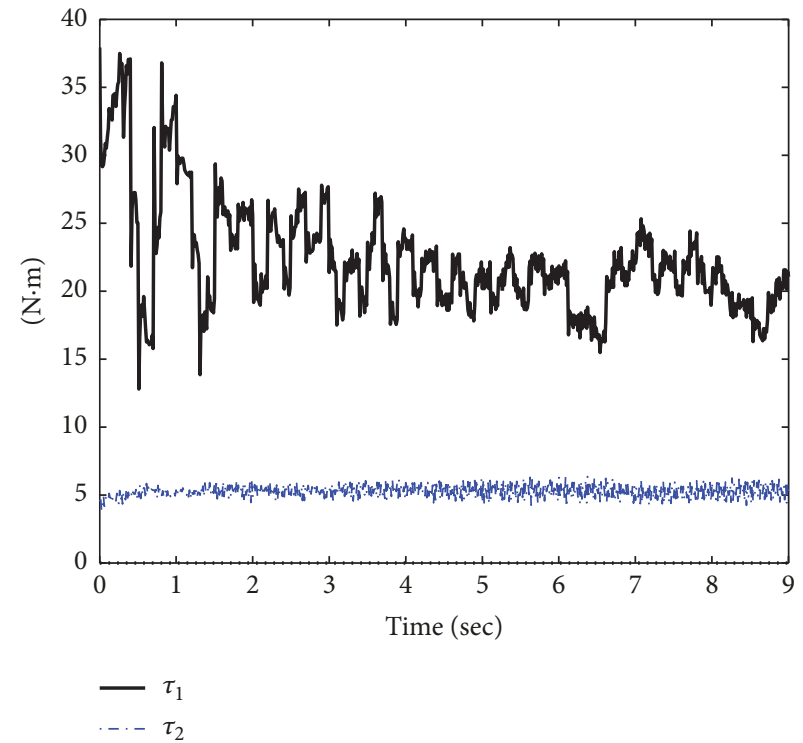

(b) Applied hyperbolic control torques with $k=1$

FIGURE 5: Experimental results of the hyperbolic control $\boldsymbol{\tau}_{1}=\mathbf{J}(\mathbf{q})^{T} \mathbf{f}_{\chi_{1}}$ for $k=1$.

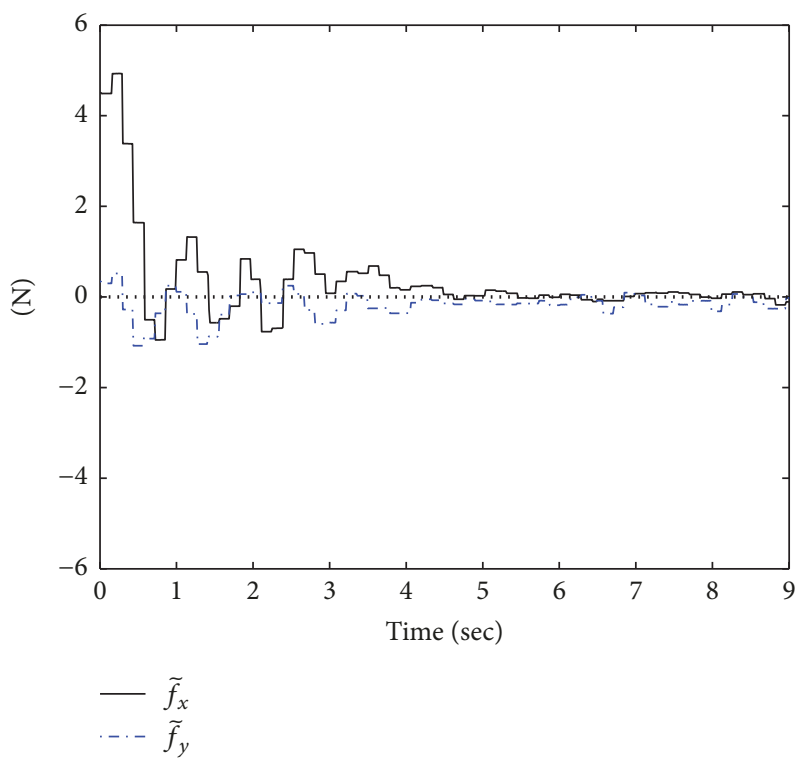

(a) Force error of the hyperbolic controller, $k=2$

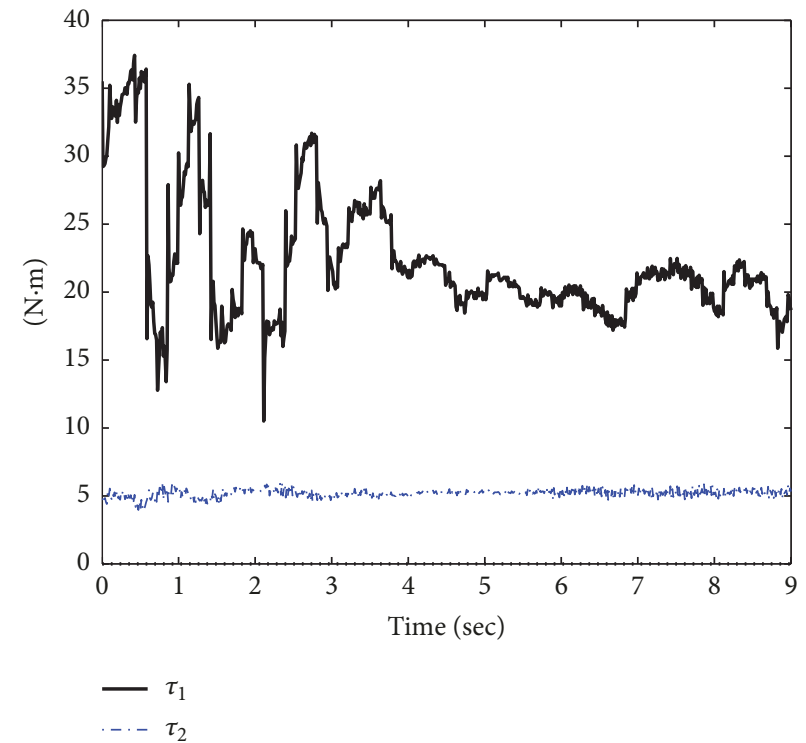

(b) Applied hyperbolic control torques, $k=2$

FIGURE 6: Experimental results for the hyperbolic control $\boldsymbol{\tau}_{2}=\mathbf{J}(\mathbf{q})^{T} \mathbf{f}_{\chi_{2}}$ with $k=2$.

$(t=9 \mathrm{sec})[-0.1,0.15]^{T} \mathrm{~N}$; the transient state $(4.5 \mathrm{sec})$ is similar to the PD control. However, it is smoother than the transient state of the PD control. The applied torques of the control $\boldsymbol{\tau}_{1}=\mathbf{J}(\mathbf{q})^{T} \mathbf{f}_{\chi_{1}}$ are presented in Figure 5(b); these signals are inside the prescribed limits.

The experimental results for the hyperbolic control scheme for $\mathbf{f}_{\chi_{2}}$ are depicted in Figure 6; the force errors $\widetilde{\mathbf{f}}$ have short transient (approximately $4.5 \mathrm{sec}$ ) and a smaller steadystate force error $(t=9 \mathrm{sec})[0.08,0.09]^{T} \mathrm{~N}$ than $\mathrm{PD}$, Atan, and Sqrt schemes as can be seen in Figure 6(a).

Observe that the transient phase is brief for all control schemes; the amplitude of the oscillation peaks is lower for the Sqrt, $\mathbf{f}_{\chi_{1}}$, and $\mathbf{f}_{\chi_{2}}$ controls compared to Atan and PD controls; and the steady-state force errors of the control 


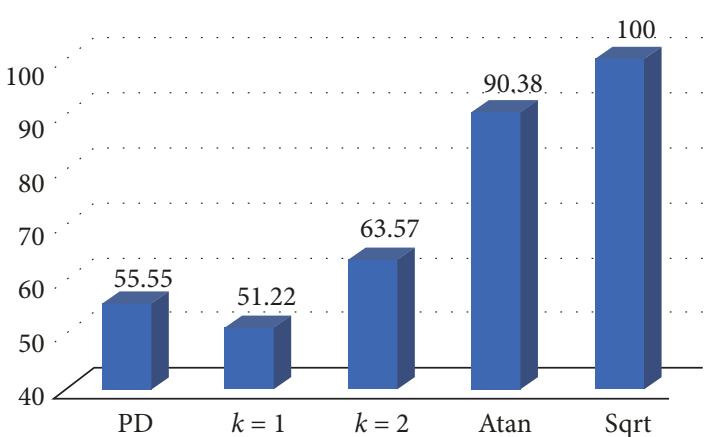

(a) Normalized $\mathscr{L}_{2}$-norm of the transitory state

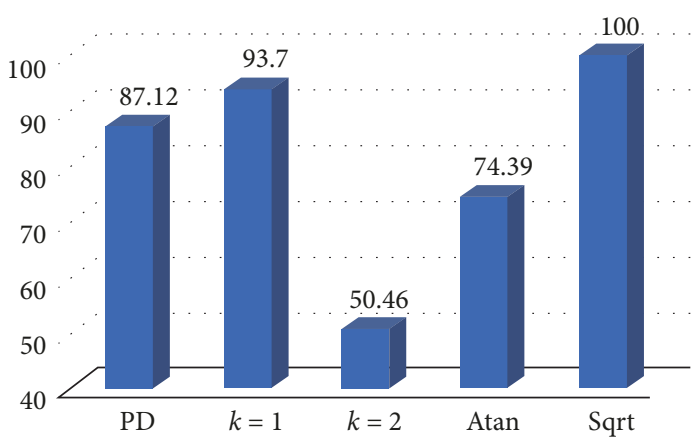

(b) Normalized $\mathscr{L}_{2}$-norm of the steady state

FIgURE 7: Performance indices $\mathscr{L}_{2}$ of evaluated control schemes.

$\mathbf{f}_{\chi_{2}}$ are smaller than PD, Atan, Sqrt, and $\mathbf{f}_{\chi_{1}}$ controls. As a consequence, the novel hyperbolic controllers yield an increased control performance for the explicit force problem of robot manipulators. Steady-state force errors are present due to the presence of static friction at joints and due to the lack of a model for this type of friction in the environment. It is important to note that, despite the presence of nonmodeled friction phenomena, the force errors are acceptably small. The results for the applied torques $\boldsymbol{\tau}_{2}=\mathbf{J}(\mathbf{q})^{T} \mathbf{f}_{\chi_{2}}$ are shown in Figure 6(b). The profile of these torque signals clearly evolves within the physical limits.

The hyperbolic-type control schemes work as follows: for large errors $\widetilde{f}_{i}$, the hyperbolic function $\cosh ^{m-1}\left(\widetilde{f}_{i}\right) \sinh \left(\widetilde{f}_{i}\right)$ $/\left(1+\cosh ^{m}\left(\widetilde{f}_{i}\right)\right)$ returns a bounded control output avoiding input saturation in the servo amplifier; when the errors $\widetilde{f}_{i}$ are small, that hyperbolic function is approximately linear; then it works like proportional controller. In contrast, with PD control, $\mathbf{f}_{\chi_{\text {PD }}}$ can demand a large amount of torque and it is not saturated. The derivative action of the Atan and Sqrt schemes is bounded, while that for the proposed hyperbolictype control is linear, demanding the effect of damping injection as needed. It is important to emphasize that, in the experiments presented herein, the applied control torques are guaranteed to be within the linear zone of the servo amplifier. Therefore, problems associated with linear control, related to vibration, thermal effects, mechanical noise, and nonmodeled dynamics, are avoided. Linear control is highly demanding in terms of gain tuning, as compared to the scheme proposed herein. In accordance with the analytical results, the proposed family of control schemes provides good performance.

In order to evaluate and compare the performances of the controllers in a scalar form, the norm $\mathscr{L}_{2}$ of the force error is used; it is an objective numerical measure of the force error $\widetilde{\mathbf{f}}$ [20]; a smaller norm $\mathscr{L}_{2}\{\tilde{\mathbf{f}}\}$ represents smaller force error and it means the best performance of the evaluated control:

$$
\mathscr{L}_{2}\{\widetilde{\mathbf{f}}\}=\sqrt{\frac{1}{T} \int_{0}^{T}\|\widetilde{\mathbf{f}}(\sigma)\|^{2} d \sigma},
$$

where $T$ is the time of the experiment. The norm $\mathscr{L}_{2}\{\widetilde{\mathbf{f}}\}$ measures the root-mean-square "average" of the force error $\widetilde{\mathbf{f}}$.
The performance comparison $\mathscr{L}_{2}\{\tilde{\mathbf{f}}\}$ of the evaluated control schemes took into account the transitory and steady states; that is, two requirements were considered on five evaluated controllers. The first one consisted of getting the performance of the transient response and the second aimed at achieving steady-state response. Once the performance was evaluated for each controller through the $\mathscr{L}_{2}\{\tilde{\mathbf{f}}\}$-norm, then the results are normalized with respect to the largest value and presented in percent scale for the transitory phase in Figure $7(\mathrm{a})$. The time interval to measure the $\mathscr{L}_{2}\{\tilde{\mathbf{f}}\}$-norm in the transitory phase is considered at $t \in[0,4.5] \mathrm{sec}$. The best performance for the transient state corresponds to the hyperbolic-type control with exponent $k=1$, corresponding to the smallest index, $51.22 \%$, with respect to the largest value of the Sqrt control. Note that, for the transitory response shown in Figure 4(a), the Euclidean norm square of the force error $\|\widetilde{\mathbf{f}}\|^{2}$ for the control $\mathbf{f}_{\chi_{\text {Sqrt }}}$ shows a larger area than other evaluated schemes; therefore a poor performance $\mathscr{L}_{2}\{\tilde{\mathbf{f}}\}$ is obtained for the Sqrt control. The PD control was the second best performance, followed by hyperbolic-type control (exponent $k=1$ ) with a good performance.

The comparative results of performance indexes for the steady state are shown in Figure $7\left(\right.$ b). The smaller $\mathscr{L}_{2}\{\widetilde{\mathbf{f}}\}$ shows that the $k=2$ hyperbolic-type control with $50.46 \%$ is the one with the best performance, because the steadyforce errors presented less error than the rest of the evaluated control algorithms; the Sqrt control obtained the largest performance index and therefore a poor performance.

The total performance index for each evaluated control scheme would be the average of the sum of indexes of the transitory and steady states; then $\mathscr{L}_{2_{\mathrm{f}_{\chi_{\mathrm{PD}}}}}=71.335, \mathscr{L}_{2_{\mathrm{f}_{\chi_{\text {Atan }}}}}=$ $82.385, \mathscr{L}_{2_{\mathrm{f}_{\chi_{\mathrm{Sqrt}}}}}=100, \mathscr{L}_{2_{\mathrm{f}_{\chi_{1}}}}=72.46$, and $\mathscr{L}_{2_{\mathrm{f}_{\chi_{2}}}}=57.015$, which correspond to $\mathrm{PD}$, Atan, Sqrt, $k=1$, and $k=2$ hyperbolictype controls, respectively. Therefore, the best performance corresponds to the hyperbolic-type control scheme $\mathbf{f}_{\chi_{2}}$.

\section{Conclusion}

In this paper, a new family of explicit force control schemes is presented. The proposed control family includes a large class of saturated-type hyperbolic function terms; the hyperbolic 
structure depends on $k$ as a power function. It includes also an active velocity damping term plus gravity compensation. Our proposal of force regulators is supported by a stability analysis in Lyapunov sense using a strict Lyapunov function. The asymptotic stability of the closed-loop system equilibrium point in Cartesian space is guaranteed. The mathematical development of Property 4 for the Cartesian dynamics model has been completed; this property is very useful for the stability analysis.

In order to show the performance of the controllers, experimental results have been obtained. An experimental comparison of the performances obtained with the classical proportional-derivative (PD), Atan, and Sqrt controls and two members of the proposed control family on a two-degree-of-freedom, direct-drive robot manipulator is presented. A force sensor (a force/torque sensor ATI FT Gamma SI-130-10) is mounted at the end-effector of the robot manipulator with the purpose of measuring the contact force in order to feed back the force error in the closedloop. The experimental evidence shows that the controller in transitory state with the best performance is the hyperbolic structure with $k=1$, followed by the hyperbolic controller with $k=2$, while in steady state the best performance corresponds to the hyperbolic control with exponent $k=$ 2. The performance of the proposed control is improved compared with traditional PD control scheme and it is a good candidate for industrial applications. The effectiveness of the proposed control scheme can be concluded.

\section{Appendix}

The Cartesian dynamic model has some useful properties for stability analysis of robot controllers. Among these properties, Property 4 is widely cited in the literature of Cartesian robot control; however, we did not find in the literature the proof that the norm of vector $\mathbf{C}_{\chi} \dot{\mathbf{x}}$ is bounded. Therefore, the mathematical development for Property 4 is presented in this section. For the validity of the Cartesian dynamic model, it is assumed that the Jacobian matrix is full rank.

Proof of Property 4. First, it is necessary to prove that $\|\dot{J}(\mathbf{q})\| \leq$ $k_{j(q)}\|\dot{\mathbf{q}}\|$, for some $k_{j(q)} \in \mathbb{R}_{+}$, is true. Since $\sqrt{\dot{q}_{i}^{2}} \leq$ $\sqrt{\dot{q}_{1}^{2}+\cdots+\dot{q}_{i}^{2}+\cdots+\dot{q}_{n}^{2}}$, then $\dot{q}_{i} \leq\|\dot{\mathbf{q}}\|$. Also,

$$
\begin{aligned}
\|\dot{j}(\mathbf{q})\| & =\left\|\frac{\partial \mathbf{J}(\mathbf{q})}{\partial q_{1}} \dot{q}_{1}+\cdots+\frac{\partial \mathbf{J}(\mathbf{q})}{\partial q_{n}} \dot{q}_{n}\right\| \\
& \leq\left\|\frac{\partial \mathbf{J}(\mathbf{q})}{\partial q_{1}} \dot{q}_{1}\right\|+\cdots+\left\|\frac{\partial \mathbf{J}(\mathbf{q})}{\partial q_{n}} \dot{q}_{n}\right\| \\
& \leq\left[\sum_{i=1}^{n}\left\|\frac{\partial \mathbf{J}(\mathbf{q})}{\partial q_{i}}\right\|\right]\|\dot{\mathbf{q}}\|=k_{j(q)}\|\dot{\mathbf{q}}\| .
\end{aligned}
$$

For the next step, consider that $\|\mathbf{C}(\mathbf{q}, \dot{\mathbf{q}}) \dot{\mathbf{q}}\| \leq k_{c 1}\|\dot{\mathbf{q}}\|^{2}$. Also, since $\dot{\mathbf{x}}=\mathbf{J}(\mathbf{q}) \dot{\mathbf{q}}$, then $\|\dot{\mathbf{q}}\| \leq\left\|J^{-1}(\mathbf{q})\right\|\|\dot{\mathbf{x}}\|$; hence,

$$
\begin{aligned}
\left\|\mathbf{C}_{\chi} \dot{\mathbf{x}}\right\| & =\left\|\mathbf{J}^{-T}(\mathbf{q}) \mathbf{C}(\mathbf{q}, \dot{\mathbf{q}}) \dot{\mathbf{q}}-\mathbf{M}_{\chi} \dot{\mathbf{J}}(\mathbf{q}) \dot{\mathbf{q}}\right\| \\
& \leq\left\|\mathbf{J}^{-1}(\mathbf{q})\right\| k_{c 1}\|\dot{\mathbf{q}}\|^{2}+\left\|\mathbf{M}_{\chi}\right\| k_{j(q)}\|\dot{\mathbf{q}}\|^{2}
\end{aligned}
$$

$$
\begin{aligned}
& \leq\left\|\mathbf{J}^{-1}(\mathbf{q})\right\|^{2}\left(\left\|\mathbf{J}^{-1}(\mathbf{q})\right\| k_{c 1}+\left\|\mathbf{M}_{\chi}\right\| k_{j(q)}\right)\|\dot{\mathbf{x}}\|^{2} \\
& =k_{c x}\|\dot{\mathbf{x}}\|^{2} .
\end{aligned}
$$

Therefore, $\left\|\mathbf{C}_{\chi} \dot{\mathbf{x}}\right\| \leq k_{c x}\|\dot{\mathbf{x}}\|^{2}$.

\section{Conflicts of Interest}

The authors declare that there are no conflicts of interest regarding this paper.

\section{Acknowledgments}

This work is partially supported by BUAP, Mexico (Project VIEP-00110).

\section{References}

[1] M. H. Raibert and J. J. Craig, "Hybrid posiition/force control of manipulators," Journal of Dynamic Systems, Measurement, and Control, vol. 103, no. 2, pp. 126-133, 1981.

[2] N. Hogan, "Impedance control - An approach to manipulation. I - Theory. II - Implementation. III - Applications," ASME Transactions Journal of Dynamic Systems and Measurement Control B, vol. 107, pp. 1-24, mar 1985.

[3] B. Siciliano and K. Oussama, Handbook of Robotics, Springer, 2nd edition, 2016.

[4] H. Kazerooni, T. B. Sheridan, and P. K. Houpt, "Robust Compliant Motion for Manipulators, Part I: The Fundamental Concepts of Compliant Motion," IEEE Journal on Robotics and Automation, vol. 2, no. 2, pp. 83-92, 1986.

[5] D. E. Whitney, "Historical Perspective and State of the Art in Robot Force Control," International Journal of Robotics Research, vol. 6, no. 1, pp. 3-14, 1987.

[6] O. Khatib, "A unified approach for motion and force control of robot manipulators: the operational space formulation," IEEE Transactions on Robotics and Automation, vol. 3, no. 1, pp. 4353, 1987.

[7] R. Carelli and R. Kelly, "An adaptive impedance/force controller for robot manipulators," Institute of Electrical and Electronics Engineers Transactions on Automatic Control, vol. 36, no. 8, pp. 967-971, 1991.

[8] S. P. Patarinski and R. G. Botev, "Robot force control: A review," Mechatronics, vol. 3, no. 4, pp. 377-398, 1993.

[9] M. Mendoza-Gutiérrez, F. Reyes, I. Bonilla-Gutiérrez, and E. González-Galván, "Proportional-derivative impedance control of robot manipulators for interaction tasks," Proceedings of the Institution of Mechanical Engineers, Part I: Journal of Systems and Control Engineering, vol. 225, no. 3, pp. 315-329, 2011.

[10] B. Siciliano and L. Villani, Robot Force Control, Kluwer Academic Publishers, Boston, MA, USA, 1999.

[11] I. Bonilla-Guti, F. Reyes, M. Mendoza-Guti, and E. J. Gonz, "A Dynamic-compensation Approach to Impedance Control of Robot Manipulators," Journal of Intelligent Robotic Systems, vol. 63, pp. 51-73, 2011.

[12] M. Mendoza, I. Bonilla, F. Reyes, and E. Gonzalez-Galvan, "A Lyapunov-based design tool of impedance controllers for robot manipulators," Kybernetika, vol. 48, no. 6, pp. 1136-1155, 2012. 
[13] S. Chiaverini, B. Siciliano, and L. Villani, "A survey of robot interaction control schemes with experimental comparison," IEEE/ASME Transactions on Mechatronics, vol. 4, no. 3, pp. 273$285,1999$.

[14] A. Denève, S. Moughamir, L. Afilal, and J. Zaytoon, "Control system design of a 3-DOF upper limbs rehabilitation robot," Computer Methods and Programs in Biomedicine, vol. 89, no. 2, pp. 202-214, 2008.

[15] H. Mehdi and O. Boubaker, "Stiffness and Impedance Control Using Lyapunov Theory for Robot-Aided Rehabilitation," International Journal of Social Robotics, vol. 4, no. 1, pp. 107-119, 2012.

[16] R. Cortesão and M. Dominici, "Robot Force Control on a Beating Heart," IEEE/ASME Transactions on Mechatronics, vol. 22, no. 4, pp. 1736-1743, 2017.

[17] L. Fortuna and G. Muscato, "A roll stabilization system for a monohull ship: modeling, identification, and adaptive control," IEEE Transactions on Control Systems Technology, vol. 4, no. 1, pp. 18-28, 1996.

[18] S. Jung and T. C. Hsia, "Robust neural force control scheme under uncertainties in robot dynamics and unknown environment," IEEE Transactions on Industrial Electronics, vol. 47, no. 2, pp. 403-412, 2000.

[19] N. Kumar, V. Panwar, N. Sukavanam, S. P. Sharma, and J.-H. Borm, "Neural network based hybrid force/position control for robot manipulators," International Journal of Precision Engineering and Manufacturing, vol. 12, no. 3, pp. 419-426, 2011.

[20] H. Chaudhary, V. Panwar, R. Prasad, and N. Sukavanam, "Adaptive neuro fuzzy based hybrid force/position control for an industrial robot manipulator," Journal of Intelligent Manufacturing, vol. 27, no. 6, pp. 1299-1308, 2016.

[21] T. Yoshikawa, T. Sugie, and M. Tanaka, "Dynamic Hybrid Position/Force Control of Robot Manipulators-Controller Design and Experiment," IEEE Journal on Robotics and Automation, vol. 4, no. 6, pp. 699-705, 1988.

[22] R. Lozano and B. Brogliato, "Adaptive hybrid force-position control for redundant manipulators," Institute of Electrical and Electronics Engineers Transactions on Automatic Control, vol. 37, no. 10, pp. 1501-1505, 1992.

[23] D. Jeon and M. Tomizuka, "Learning Hybrid Force and Position Control of Robot Manipulators," IEEE Transactions on Robotics and Automation, vol. 9, no. 4, pp. 423-431, 1993.

[24] R. Volpe and P. Khosla, "A theoretical and experimental investigation of explicit force control strategies for manipulators," Institute of Electrical and Electronics Engineers Transactions on Automatic Control, vol. 38, no. 11, pp. 1634-1650, 1993.

[25] S. Chiaverini and L. Sciavicco, "Parallel approach to force/position control of robotic manipulators," IEEE Transactions on Robotics and Automation, vol. 9, no. 4, pp. 361-373, 1993.

[26] S. K. Singh and D. O. Popa, "An Analysis of Some Fundamental Problems in Adaptive Control of Force and Impedance Behavior: Theory and Experiments," IEEE Transactions on Robotics and Automation, vol. 11, no. 6, pp. 912-921, 1995.

[27] G. Ferretti, G. Magnani, and P. Rocco, "Toward the implementation of hybrid position/force control in industrial robots," IEEE Transactions on Robotics and Automation, vol. 13, no. 6, pp. 838845, 1997.

[28] G. Zeng and A. Hemami, "An overview of robot force control," Robotica, vol. 15, no. 5, pp. 473-482, 1997.

[29] C. Natale, Interaction Control of Robot Manipulators: Sixdegrees-of-freedom Tasks, Springer Tracts in Advanced Robotics, Springer, 2003.
[30] Q. H. Xia, S. Y. Lim, M. H. Ang Jr., and T. M. Lim, "Parallel force and motion control using adaptive observer-controller," in Proceedings of the 2008 IEEE International Conference on Systems, Man and Cybernetics, SMC 2008, pp. 3143-3149, Singapore, October 2008.

[31] Y. Karayiannidis and Z. Doulgeri, "Robot force/position tracking on a surface of unknown orientation," in European Robotics Symposium 2008, vol. 44 of Springer Tracts Adv. Robot., pp. 253262, Springer, Berlin, 2008.

[32] B. Siciliano, L. Sciavicco, L. Villani, and G. Oriolo, Robotics: Modelling, Planning and Control, Springer Publishing Company, Incorporated, 2010.

[33] S. A. M. Dehghan, M. Danesh, and F. Sheikholeslam, "Adaptive hybrid force/position control of robot manipulators using an adaptive force estimator in the presence of parametric uncertainty," Advanced Robotics, vol. 29, no. 4, pp. 209-223, 2015.

[34] X. Sheng, L. Xu, and Z. Wang, "A position-based explicit force control strategy based on online trajectory prediction," International Journal of Robotics and Automation, vol. 32, no. 1, pp. 93-100, 2017

[35] S. M. Hashemi, U. Gürcüoğlu, and H. Werner, "Interaction control of an industrial manipulator using LPV techniques," Mechatronics, vol. 23, no. 6, pp. 689-699, 2013.

[36] H. P. Singh and N. Sukavanam, "Stability analysis of robust adaptive hybrid position/force controller for robot manipulators using neural network with uncertainties," Neural Computing and Applications, vol. 22, no. 7-8, pp. 1745-1755, 2013.

[37] E. Sariyildiz and K. Ohnishi, "On the explicit robust force control via disturbance observer," IEEE Transactions on Industrial Electronics, vol. 62, no. 3, pp. 1581-1589, 2015.

[38] C. A. Chavez-Olivares, F. Reyes-Cortes, and E. J. GonzalezGalvan, "On Explicit Force Regulation with Active Velocity Damping for Robot Manipulators," Automatika, vol. 56, no. 4, pp. 478-490, 2015.

[39] R. D. J. Portillo-Vélez, A. Rodriguez-Angeles, and C. A. CruzVillar, "An optimization-based impedance approach for robot force regulation with prescribed force limits," Mathematical Problems in Engineering, vol. 2015, Article ID 918301, 13 pages, 2015.

[40] A. Calanca, R. Muradore, and P. Fiorini, "A review of algorithms for compliant control of stiff and fixed-compliance robots," IEEE/ASME Transactions on Mechatronics, vol. 21, no. 2, pp. 613-624, 2016.

[41] Q. Wu, X. Wang, B. Chen, H. Wu, and Z. Shao, "Development and hybrid force/position control of a compliant rescue manipulator," Mechatronics, vol. 46, pp. 143-153, 2017.

[42] J. L. Dideriksen, D. F. Feeney, A. M. Almuklass, and R. M. Enoka, "Control of force during rapid visuomotor forcematching tasks can be described by discrete time PID control algorithms," Experimental Brain Research, vol. 235, no. 8, pp. 2561-2573, 2017.

[43] P. Gierlak and M. Szuster, "Adaptive position/force control for robot manipulator in contact with a flexible environment," Robotics and Autonomous Systems, vol. 95, pp. 80-101, 2017.

[44] M. Takegaki and S. Arimoto, "New feedback method for dynamic control of manipulators," Journal of Dynamic Systems, Measurement and Control, vol. 103, no. 2, pp. 119-125, 1981.

[45] C. Ott, Cartesian Impedance Control of Redundant and Flexible Joint Robots, Springer, 2008.

[46] C. A. Chávez-Olivares, F. Reyes-Cortés, E. J. González-Galván, M. O. Mendoza-Gutiérrez, and I. Bonilla-Gutiérrez, "Experimental evaluation of parameter identification schemes on a 
direct-drive robot," Proceedings of the Institution of Mechanical Engineers, Part I: Journal of Systems and Control Engineering, vol. 226, no. 10, pp. 1419-1431, 2012.

[47] R. Kelly, V. Santibanez, and F. Reyes, "On saturated-proportional derivative feedback with adaptive gravity compensation of robot manipulators," International Journal of Adaptive Control and Signal Processing, vol. 10, no. 4-5, pp. 465-479, 1996. 


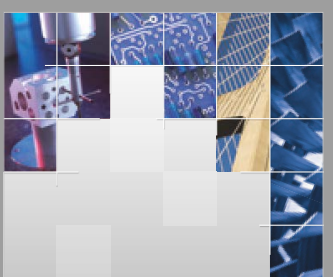

\section{Enfincering}
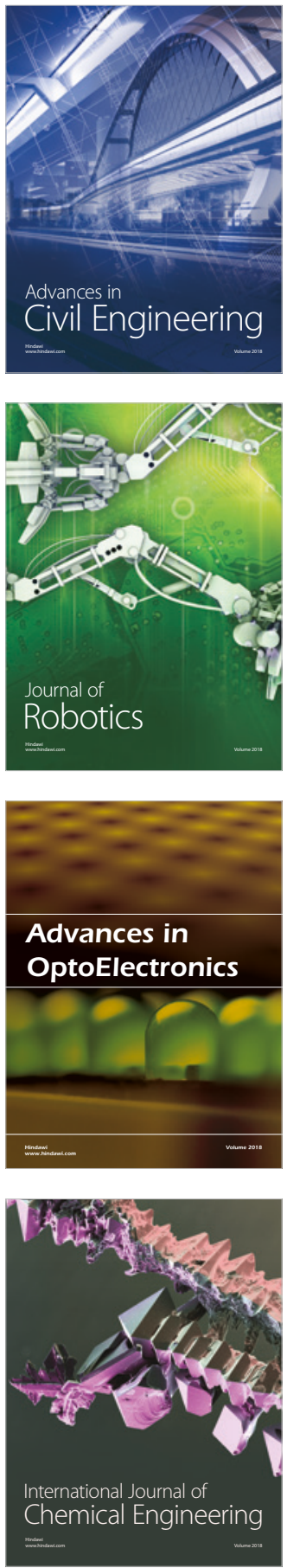

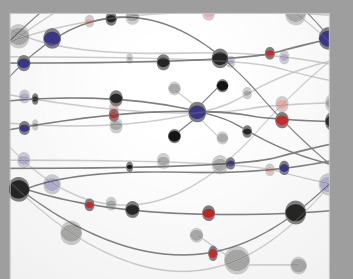

\section{Rotating \\ Machinery}

The Scientific World Journal

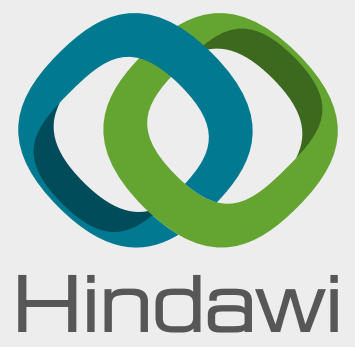

Submit your manuscripts at

www.hindawi.com
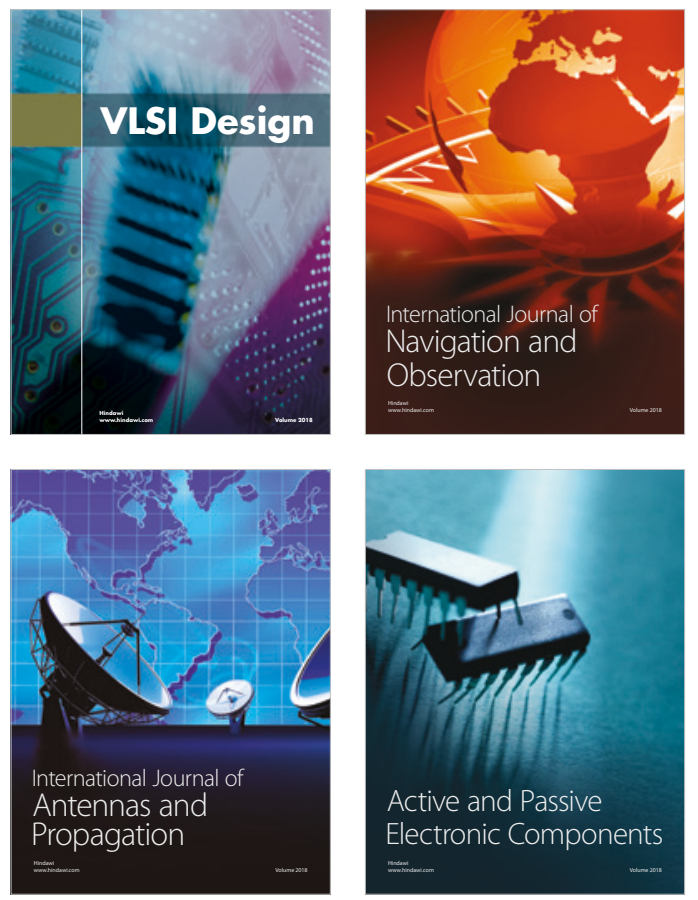
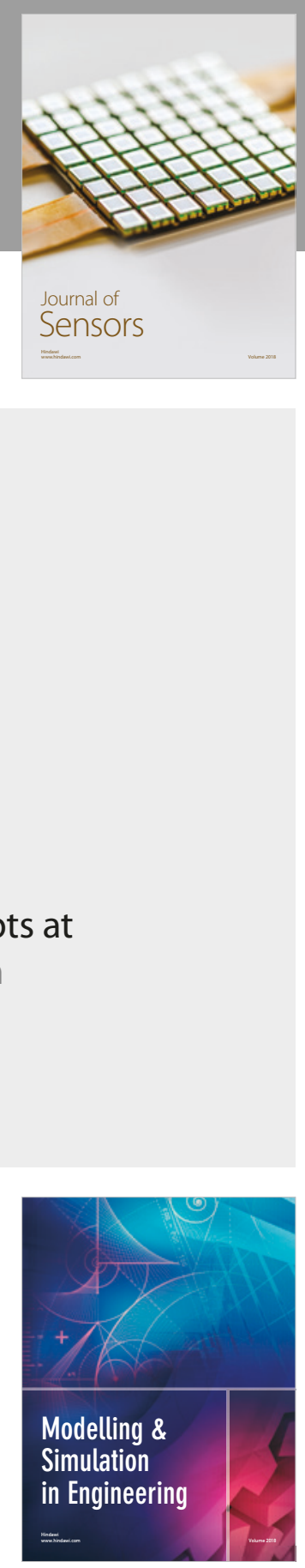

\section{Advances \\ Multimedia}
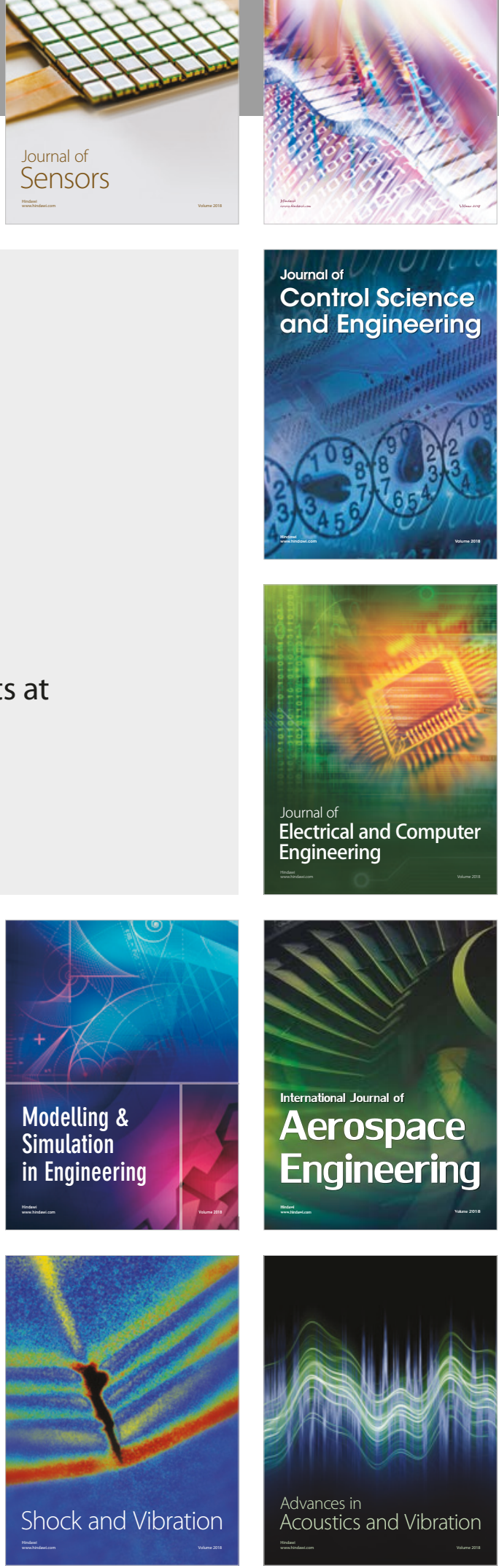